\title{
ON SMOOTH FUZZY SUBSPACES
}

\author{
S. E. ABBAS
}

Received 5 January 2004

\begin{abstract}
We introduce a new concept of smooth topological subspaces, which coincides with the usual definition in the case where $\mu=\chi_{Y}, Y \subset X$. Also, we introduce some concepts such as $q$-nbd systems, continuity, separation axioms, compactness, and connectedness in this sense. Also, various characterization for some fuzzy topological concepts in this sense are given.
\end{abstract}

2000 Mathematics Subject Classification: 54A40.

1. Introduction and preliminaries. The concept of fuzzy topology was first defined in 1968 by Chang [2] and later redefined in somewhat different way by Lowen [8] and Hutton [7]. According to Šostak [11], these definitions, a fuzzy topology is a crisp subfamily of family of fuzzy sets and fuzziness in the concept of openness of a fuzzy set has not been considered, which seems to be a drawback in the process of fuzzification of the concept of topological spaces. Therefore, Šostak introduced a new definition of fuzzy topology in 1985 [11], which we will call "smooth topology." Later on he has developed the theory of smooth topological spaces in [11, 12]. After that, several authors $[1,3,4,5,6,10]$ have reintroduced the same definition and studied smooth topological spaces being unaware of Šostak's work. They referred to the fuzzy topology in the sense of Chang as the topology of fuzzy subsets.

Throughout this paper, let $X$ be a nonempty set, $I=[0,1], I_{\circ}=(0,1]$, and $I_{1}=[0,1)$. For $\alpha \in I, \bar{\alpha}(x)=\alpha$ for all $x \in X$. The family of all fuzzy sets on $X$ is defined by $I^{X}$. For $x \in X$ and $t \in I_{\circ}$, a fuzzy point $x_{t}$ is defined by

$$
x_{t}(y)= \begin{cases}t & \text { if } y=x \\ 0 & \text { if } y \neq x\end{cases}
$$

A fuzzy point $x_{t}$ is said to be quasicoincident with the fuzzy set $U$ with respect to $\mu \in I^{X}$ if and only if $t+U(x)>\mu(x)$. We write this as $x_{t} q U[\mu]$. For $U, V \in I^{X}, U$ is quasicoincident with $V$ with respect to $\mu$. We denote this as $U q V[\mu]$, if there exists $x \in X$ such that $U(x)+V(x)>\mu(x)$. Otherwise we denote the case as $U \not V[\mu]$.

Let $(X, T)$ be a Chang fuzzy topological space and $x_{t} \in \mu$. Then we say that $V \in \mathscr{A}_{\mu}$ is a fuzzy $\mu$-q-nbd of $x_{t}$ if there is $U \in T_{\mu}$ such that $x_{t} q U[\mu]$ and $U \leq V$ [13].

A smooth topological space (STS) $[10,11]$ is an ordered pair $(X, \mathscr{T})$, where $X$ is a nonempty set and $\mathscr{T}: I^{X} \rightarrow I$ is a mapping satisfying the following conditions:

(O1) $\mathscr{T}(\overline{0})=\mathscr{T}(\overline{1})=1$;

(O2) for all $A, B \in I^{X}, \mathscr{T}(A \wedge B) \geq \mathscr{T}(A) \wedge \mathscr{T}(B)$; 
(O3) for every subfamily $\left\{A_{i}: i \in J\right\} \subseteq I^{X}, \mathscr{T}\left(\bigvee_{i \in J} A_{i}\right) \geq \bigwedge_{i \in J} \mathscr{T}\left(A_{i}\right)$.

The number $\mathscr{T}(A)$ is called the degree of openness of $A$.

Let $(X, \mathcal{T})$ be an STS and $Y \subseteq X$. Then the mapping $\mathscr{T}_{Y}: I^{Y} \rightarrow I$ defined by

$$
\mathscr{T}_{Y}(U)=\bigvee\left\{\mathscr{T}(V): V \in I^{X},\left.V\right|_{Y}=U\right\}
$$

is the induced smooth topology on $Y$ from $\mathscr{T}$, and $\left(Y, \mathscr{T}_{Y}\right)$ is a subspace of $(X, \mathscr{T})[10,11]$.

Let $(X, \mathscr{T})$ and $\left(Y, \mathscr{T}^{*}\right)$ be two STSs. A mapping $f: X \rightarrow Y$ is called fuzzy continuous $[10,11]$ if and only if $\mathscr{T}\left(f^{-1}(A)\right) \geq \mathscr{T}^{*}(A)$ for every $A \in I^{Y}$.

2. Smooth topological subspaces. For $\mu \in I^{X}$ we call $\mathscr{A}_{\mu}=\left\{U \in I^{X}: U \leq \mu\right\}$.

DEFINITION 2.1. Let $(X, \mathscr{T})$ be an STS and $\mu \in I^{X}$. The mapping $\mathscr{T}_{\mu}: \mathscr{A}_{\mu} \rightarrow I$ defined by

$$
\mathscr{T}_{\mu}(U)=\bigvee\left\{\mathscr{T}(V): V \in I^{X}, V \wedge \mu=U\right\}
$$

is a smooth $\mu$-topology induced over $\mu$ by $\mathscr{T}$. For any $U \in \mathscr{A}_{\mu}$, the number $\mathscr{T}_{\mu}(U)$ is called the $\mu$-openness degree of $U$.

It is easy to show that the above definition makes sense and to prove the following theorems.

THEOREM 2.2. $\mathscr{T}_{\mu}$ verifies the following properties:

$(\mu \mathrm{O} 1) \mathscr{T}_{\mu}(\overline{0})=\mathscr{T}_{\mu}(\mu)=1$;

$(\mu \mathrm{O} 2)$ for all $A, B \in \mathscr{A}_{\mu}, \mathscr{T}_{\mu}(A \wedge B) \geq \mathscr{T}_{\mu}(A) \wedge \mathscr{T}_{\mu}(B)$;

( $\mu$ O3) for every subfamily $\left\{A_{i}: i \in J\right\} \subseteq \mathscr{A}_{\mu}, \mathscr{T}_{\mu}\left(\bigvee_{i \in J} A_{i}\right) \geq \bigwedge_{i \in J} \mathscr{T}_{\mu}\left(A_{i}\right)$.

REMARK 2.3. If $Y \subset X$ and $\mu=\chi_{Y}$, we just have the usual concept of smooth subspace. Given $\mathscr{T}_{\mu}$ and $\nu \in \mathscr{A}_{\mu}$ we can define $\left(\mathscr{T}_{\mu}\right)_{\nu}$, the smooth $\nu$-topology induced over $\nu$ by $\mathscr{T}_{\mu}$, in the obvious way. We have trivially $\mathscr{T}_{\nu}=\left(\mathscr{T}_{\mu}\right)_{\nu}$, that is, a smooth subspace of a smooth subspace is also a smooth subspace.

REMARK 2.4. (1) Let $(X, \mathscr{T})$ be an STS and $\mu \in I^{X}$. Then, for each $\alpha \in I_{\circ}, \mathscr{T}_{\mu}^{\alpha}=\{U \in$ $\left.\mathscr{A}_{\mu}: \mathscr{T}_{\mu}(U) \geq \alpha\right\}$ is the fuzzy $\mu$-topology in the sense of Macho Stadler and De Prada Vicente [9]. Moreover, $\alpha_{1} \leq \alpha_{2}$ implies $\mathscr{T}_{\mu}^{\alpha_{1}} \geq \mathscr{T}_{\mu}^{\alpha_{2}}$. Also, $\mathscr{T}_{\mu}(A)=\sup \left\{\alpha: A \in \mathscr{T}_{\mu}^{\alpha}\right\}$ is a smooth $\mu$-topology.

(2) From a Chang fuzzy topological space $\left(X, \mathscr{T}_{\mu}^{\alpha}\right)$, we can identify a smooth $\mu$ topology $\mathscr{T}_{\alpha \mu}: \mathscr{A}_{\mu} \rightarrow I$,

$$
\mathscr{T}_{\alpha \mu}(A)= \begin{cases}1 & \text { if } A \in \mathscr{T}_{\mu}^{\alpha}, \\ 0 & \text { if } A \notin \mathscr{T}_{\mu}^{\alpha},\end{cases}
$$

for each $A \in \mathscr{A}_{\mu}$.

THEOREM 2.5. Let $(X, \mathcal{T})$ be an STS and $\mu \in I^{X}$. Then, for each $\alpha \in I_{\circ}, U \in \mathscr{A}_{\mu}$, define an operator $\mathrm{Cl}_{\mu}: \mathscr{A}_{\mu} \times I_{\circ} \rightarrow \mathscr{A}_{\mu}$ as follows:

$$
\mathrm{Cl}_{\mu}(U, \alpha)=\bigwedge\left\{V \in \mathscr{A}_{\mu}: U \leq V, \mathscr{T}_{\mu}(\mu-V) \geq \alpha\right\}
$$


For $U_{1}, U_{2} \in \mathscr{A}_{\mu}$ and $\alpha, \beta \in I_{\circ}$, the operator $\mathrm{Cl}_{\mu}$ satisfies the following conditions:

$(\mu \mathrm{C} 1) \mathrm{Cl}_{\mu}(\overline{0}, \alpha)=\overline{0}$;

$(\mu \mathrm{C} 2) U_{1} \leq \mathrm{Cl}_{\mu}\left(U_{1}, \alpha\right)$;

$(\mu \mathrm{C} 3) \mathrm{Cl}_{\mu}\left(U_{1}, \alpha\right) \vee \mathrm{Cl}_{\mu}\left(U_{2}, \alpha\right)=\mathrm{Cl}_{\mu}\left(U_{1} \vee U_{2}, \alpha\right)$;

( $\mu$ C4) $\mathrm{Cl}_{\mu}\left(U_{1}, \alpha\right) \leq \mathrm{Cl}_{\mu}\left(U_{1}, \beta\right)$ if $\alpha \leq \beta$;

( $\mu$ C5) $\mathrm{Cl}_{\mu}\left(\mathrm{Cl}_{\mu}\left(U_{1}, \alpha\right), \alpha\right)=\mathrm{Cl}_{\mu}\left(U_{1}, \alpha\right)$.

THEOREM 2.6. Let $(X, \mathcal{T})$ be an STS and $\mu \in I^{X}$. Then, for each $\alpha \in I_{\circ}, U \in \mathscr{A}_{\mu}$, define an operator $\operatorname{Int}_{\mu}: \mathscr{A}_{\mu} \times I_{\circ} \rightarrow \mathscr{A}_{\mu}$ as follows:

$$
\operatorname{Int}_{\mu}(U, \alpha)=\bigvee\left\{V \in \mathscr{A}_{\mu}: U \geq V, \mathscr{T}_{\mu}(V) \geq \alpha\right\}
$$

For $U_{1}, U_{2} \in \mathscr{A}_{\mu}$ and $\alpha, \beta \in I_{\circ}$, the operator $\operatorname{Int}_{\mu}$ satisfies the following conditions:

( $\mu \mathrm{I} 1) \operatorname{Int}_{\mu}\left(\mu-U_{1}, \alpha\right)=\mu-\mathrm{Cl}_{\mu}\left(U_{1}, \alpha\right)$ and $\mathrm{Cl}_{\mu}\left(\mu-U_{1}, \alpha\right)=\mu-\operatorname{Int}_{\mu}\left(U_{1}, \alpha\right)$;

$(\mu \mathrm{I} 2) \operatorname{Int}_{\mu}(\mu, \alpha)=\mu$;

$(\mu \mathrm{I} 3) \operatorname{Int}_{\mu}\left(U_{1}, \alpha\right) \leq U_{1}$;

$(\mu \mathrm{I} 4) \operatorname{Int}_{\mu}\left(U_{1}, \alpha\right) \wedge \operatorname{Int}_{\mu}\left(U_{2}, \alpha\right)=\operatorname{Int}_{\mu}\left(U_{1} \wedge U_{2}, \alpha\right)$;

$(\mu \mathrm{I} 5) \operatorname{Int}_{\mu}\left(U_{1}, \alpha\right) \geq \operatorname{Int}_{\mu}\left(U_{1}, \beta\right)$ if $\alpha \leq \beta$;

( $\mu$ I6) $\operatorname{Int}_{\mu}\left(\operatorname{Int}_{\mu}\left(U_{1}, \alpha\right), \alpha\right)=\operatorname{Int}_{\mu}\left(U_{1}, \alpha\right)$.

THEOREM 2.7. Let $(X, \mathcal{T})$ be an STS, $\alpha \in I_{\circ}, \mu \in I^{X}, x_{t} \in \mu$, and $U \in \mathscr{A}_{\mu}$. Then $x_{t} \in$ $\mathrm{Cl}_{\mu}(U, \alpha)$ if and only if for each $V \in \mathscr{A}_{\mu}$ such that $\mathscr{T}_{\mu}(V) \geq \alpha$ and $x_{t} q V[\mu], U q V[\mu]$ holds.

Proof. Let $x_{t} \in \mathrm{Cl}_{\mu}(U, \alpha), V \in \mathscr{A}_{\mu}$ such that $\mathscr{T}_{\mu}(V) \geq \alpha, x_{t} q V[\mu]$. Suppose that $U \not V[\mu]$ which implies $U \leq \mu-V$. From $x_{t} q V[\mu]$ we have $x_{t} \notin \mu-V \geq U$. Since $\mathscr{T}_{\mu}(\mu-$ $(\mu-V)) \geq \alpha$, then $x_{t} \notin \mathrm{Cl}_{\mu}(U, \alpha)$ which is a contradiction. Hence $U q V[\mu]$.

Conversely, let $V \in \mathscr{A}_{\mu}$ such that $\mathscr{T}_{\mu}(V) \geq \alpha, x_{t} q V[\mu]$, and $U q V[\mu]$. Suppose that $x_{t} \notin \mathrm{Cl}_{\mu}(U, \alpha)$. Then there is $W \in \mathscr{A}_{\mu}$ such that $\mathscr{T}_{\mu}(\mu-W) \geq \alpha, W \geq U$, and $x_{t} \notin W$. From $x_{t} \notin W$ we have $x_{t} q(\mu-W)[\mu]$. Then, from our hypotheses $U q(\mu-W)[\mu]$ which implies that $U \npreceq W$. This is a contradiction. Hence $x_{t} \in \mathrm{Cl}_{\mu}(U, \alpha)$.

3. Fuzzy $\mu$-q-neighborhood systems. Here we build a fuzzy $\mu$-q-neighborhood system of a fuzzy set in an STS and we introduce some of its properties. For a mapping 2: $\mathscr{A}_{\mu} \rightarrow I^{\mathscr{A}_{\mu}}, A \in \mathscr{A}_{\mu}$, and $\alpha \in I_{1}$, we define the family $2_{A}^{\alpha}=\left\{B \in \mathscr{A}_{\mu}: \mathscr{2}_{A}(B)=2(A)(B)>\alpha\right\}$, which will play an important role in this section.

DeFINITION 3.1. Let $(X, \mathscr{T})$ be an STS, $\mu \in I^{X}$, and $A \in \mathscr{A}_{\mu}$. Then the mapping 2 : $\mathscr{A}_{\mu} \rightarrow I^{\lrcorner_{\mu}}$ is called the fuzzy $\mu$-q-neighborhood ( $\mu$-q-nbd, for short) of $A$ with respect to $\mathscr{T}_{\mu}$ if and only if for each $\alpha \in I_{1}$,

$$
2_{A}^{\alpha}=\left\{B \in \mathscr{A}_{\mu}:\left(\exists C \in \mathscr{A}_{\mu}: \mathscr{T}_{\mu}(C) \geq \alpha\right)(A q C[\mu] \leq B)\right\}
$$

REMARK 3.2. The real number $2_{A}(B)$ is called the degree of $\mu$ - $q$-nbdness of the fuzzy set $B$ to the fuzzy set $A$. If the fuzzy $\mu$-q-nbd system of a fuzzy set $A$ has the following property: $\mathscr{2}_{A}\left(\mathscr{A}_{\mu}\right) \subseteq\{0,1\}$, then $\mathscr{2}_{A}$ is called the $\mu$-q-nbd system of $A$ (given by Zahran [13]). 
THEOREM 3.3. Let $(X, \mathscr{T})$ be an $S T S, \mu \in I^{X}$, and $A \in A_{\mu}$. Then the mapping $2_{A}: \mathscr{A}_{\mu} \rightarrow I$ is the fuzzy $\mu-q-n b d$ system of $A$ with respect to the $\mathscr{T}_{\mu}$ if and only if

$$
\mathscr{Q}_{A}(B)= \begin{cases}\sup \left\{\mathscr{T}_{\mu}(C): C \in \mathscr{A}_{\mu}, A q C[\mu] \leq B\right\}, & A q B[\mu], \\ 0, & A \not \notin B[\mu] .\end{cases}
$$

Proof. "If" part. Suppose that the mapping $\mathscr{2}_{A}: \mathscr{A}_{\mu} \rightarrow I$ is the fuzzy $\mu$ - $q$-nbd system of $A$ with respect to $\mathscr{T}_{\mu}$ and consider the following cases.

(a) For the case $A \not B[\mu]$, suppose that $2_{A}(B)>0$. From Definition 3.1, there exists $C \in \mathscr{A}_{\mu}$ with $\mathscr{T}_{\mu}(A) \geq \alpha$ for all $\alpha \in I_{\circ}$ such that $A q C[\mu] \leq B$, that is, $A q B[\mu]$ is a contradiction. Thus, $\mathscr{2}_{A}(B)=0$.

(b) For the case $A q B[\mu]$, we may have $\mathscr{2}_{A}(B)=0$ or $\mathscr{2}_{A}(B)>0$. If $\mathscr{2}_{A}(B)=0$, then it is obvious that $\mathscr{2}_{A}(B)=0 \leq \sup \left\{\mathscr{T}_{\mu}(C): C \in \mathscr{A}_{\mu}, A q C[\mu] \leq B\right\}$; if $\sup \left\{\mathscr{T}_{\mu}(C): C \in\right.$ $\left.\mathscr{A}_{\mu}, A q C[\mu] \leq B\right\}=s>o$, then there exists $C \in \mathscr{A}_{\mu}$ such that $\mathscr{T}_{\mu}(C)>0$ and $A q C[\mu] \leq$ $B$. We obtain $2_{A}(B)>0$, which is a contradiction. Therefore,

$$
\mathscr{2}_{A}(B)=0=\sup \left\{\mathscr{T}_{\mu}(C): C \in \mathscr{A}_{\mu}, A q C[\mu] \leq B\right\}
$$

Now suppose that $\mathscr{2}_{A}(B)=s>0$. For an arbitrary $0<\epsilon \leq s$, we have $\mathscr{2}_{A}(B)>s-\epsilon$, that is, $B \in 2_{A}^{s-\epsilon}$. Since the mapping $\mathscr{2}_{A}: \mathscr{A}_{\mu} \rightarrow I$ is a fuzzy $\mu$ - $q$-nbd system of $A$, there exists $C \in \mathscr{A}_{\mu}$ with $\mathscr{T}_{\mu}(C) \geq s-\epsilon$ and $A q C[\mu] \leq B$, that is, $\sup \left\{\mathscr{T}_{\mu}(C): C \in \mathscr{A}_{\mu}, A q C[\mu] \leq\right.$ $B\}>s-\epsilon$. Since $\epsilon>0$ is arbitrary, we have

$$
\sup \left\{\mathscr{T}_{\mu}(C): C \in \mathscr{A}_{\mu}, A q C[\mu] \leq B\right\} \geq s=\mathscr{2}_{A}(B)
$$

On the other hand, let $\sup \left\{\mathscr{T}_{\mu}(C): C \in \mathscr{A}_{\mu}, A q C[\mu] \leq B\right\}=n>0$. Then for every $0<\epsilon \leq n$, there exists $C \in \mathscr{A}_{\mu}$ such that $\mathscr{T}_{\mu}(C)>n-\epsilon$ and $A q C[\mu] \leq B$. Therefore $B \in 2_{A}^{n-\epsilon}$, that is, $\mathscr{2}_{A}(B) \geq n-\epsilon$. Since $\epsilon$ is arbitrary we have

$$
\mathscr{2}_{A}(B) \geq n=\sup \left\{\mathscr{T}_{\mu}(C): C \in \mathscr{A}_{\mu}, A q C[\mu] \leq B\right\} .
$$

Hence the inequality follows.

"Only if" part. For $\alpha \in I_{1}$, let $B \in 2_{A}^{\alpha}$, that is, $\mathscr{2}_{A}(B) \geq \alpha$. Then we can write $\alpha \leq$ $\mathscr{2}_{A}(B)=\sup \left\{\mathscr{T}_{\mu}(C): C \in \mathscr{A}_{\mu}, A q C[\mu] \leq B\right\}$. Then we have

$$
2_{A}^{\alpha} \subseteq\left\{B \in \mathscr{A}_{\mu}:\left(\exists C \in \mathscr{A}_{\mu}: \mathscr{T}_{\mu}(C) \geq \alpha\right)(A q C[\mu] \leq B)\right\}
$$

By the same way we can show that

$$
\left\{B \in \mathscr{A}_{\mu}:\left(\exists C \in \mathscr{A}_{\mu}: \mathscr{T}_{\mu}(C) \geq \alpha\right)(A q C[\mu] \leq B)\right\} \subseteq \mathscr{2}_{A}^{\alpha}
$$

Hence, $\mathscr{2}_{A}^{\alpha}=\left\{B \in \mathscr{A}_{\mu}:\left(\exists C \in \mathscr{A}_{\mu}\right.\right.$ such that $\left.\left.\mathscr{T}_{\mu}(C) \geq \alpha\right)(A q C[\mu] \leq B)\right\}$. 
REMARK 3.4. In Theorem 3.3, the fuzzy subset $A$ of $X$ can be replaced by the fuzzy point on $X$, that is, by the special fuzzy subsets $x_{t}$. In this case,

$$
\mathscr{2}_{x_{t}}(B)= \begin{cases}\sup \left\{\mathscr{T}_{\mu}(C): C \in \mathcal{A}_{\mu}, x_{t} q C[\mu] \leq B\right\}, & x_{t} q B[\mu], \\ 0, & x_{t} \phi B[\mu] .\end{cases}
$$

THEOREM 3.5. Let $(X, \mathscr{T})$ be an STS, $\mu \in I^{X}$, and $A \in \mathcal{A}_{\mu}$. If the mapping $2_{A}: \mathscr{A}_{\mu} \rightarrow I$ is the fuzzy $\mu-q$-nbd system of $A$ with respect to $\mathscr{T}_{\mu}$, then the following properties hold: $(\mu \mathrm{Q} 1) 2_{\overline{0}}(\overline{0})=\mathscr{2}_{\mu}(\mu)=1$ and $\mathscr{2}_{A}(B)>0$ implies $A \leq B$;

( $\mu$ Q2) if $A_{1} \leq A$ and $B \leq B_{1}$, then $2_{A}(B) \leq 2_{A_{1}}\left(B_{1}\right)$;

$(\mu \mathrm{Q} 3) \mathscr{2}_{A}\left(B_{1}\right) \wedge 2_{A}\left(B_{2}\right) \leq 2_{A}\left(B_{1} \wedge B_{2}\right)$;

$(\mu \mathrm{Q} 4) \mathscr{2}_{A}(B) \leq \sup _{A q C[\mu] \leq B}\left\{\mathscr{2}_{A}(C) \wedge \mathscr{2}_{C}(B)\right\}$, for all $A, B \in \mathscr{A}_{\mu}$;

$(\mu \mathrm{Q} 5) \sup \left\{\mathscr{Q}_{A}(U): U \in \mathscr{A}_{\mu}\right\}=1$.

Proof. ( $\mu \mathrm{Q} 1),(\mu \mathrm{Q} 2)$, and ( $\mu \mathrm{Q} 5)$ follow directly from Definition 3.1 and Theorem 3.3.

( $\mu$ Q3) Suppose that $\mathscr{2}_{A}\left(B_{1}\right)=m>0$ and $\mathscr{2}_{A}\left(B_{2}\right)=n>0$. Then for a fixed $\epsilon>0$ such that $\epsilon \leq m \wedge n$ implies $\mathscr{2}_{A}\left(B_{1}\right)>m-\epsilon \geq 0$ and $\mathscr{2}_{A}\left(B_{2}\right)>n-\epsilon \geq 0$. From Definition 3.1, it is clear that there exists $C_{1}, C_{2} \in \mathscr{A}_{\mu}$ such that $\mathscr{T}_{\mu}\left(C_{1}\right)>m-\epsilon, \mathscr{T}_{\mu}\left(C_{2}\right)>n-\epsilon$ and $A q C_{1}[\mu] \leq B_{1}, A q C_{2}[\mu] \leq B_{2}$. Therefore, $\mathscr{T}_{\mu}\left(C_{1} \wedge C_{2}\right) \geq \mathscr{T}_{\mu}\left(C_{1}\right) \wedge \mathscr{T}_{\mu}\left(C_{2}\right)>(m-\epsilon) \wedge$ $(n-\epsilon)=(m \wedge n)-\epsilon$ and $A q\left(C_{1} \wedge C_{2}\right)[\mu] \leq B_{1} \wedge B_{2}$. Thus, $2_{A}\left(B_{1} \wedge B_{2}\right) \geq(m \wedge n)-\epsilon$. Since $\epsilon$ is arbitrary, we find that

$$
\mathscr{2}_{A}\left(B_{1} \wedge B_{2}\right) \geq \mathscr{2}_{A}\left(B_{1}\right) \wedge \mathscr{2}_{A}\left(B_{2}\right) .
$$

$(\mu \mathrm{Q} 4) \mathscr{2}_{A}(B)=\sup \left\{\mathscr{T}_{\mu}(C): C \in \mathscr{A}_{\mu}, A q C[\mu] \leq B\right\}$. From Theorem 3.3, we obtain $\mathscr{T}_{\mu}(C) \leq \mathscr{2}_{A}(C)$ and $\mathscr{T}_{\mu}(C) \leq \mathscr{2}_{C}(B)$. Thus,

$$
\sup \left\{\mathscr{T}_{\mu}(C): C \in \mathscr{A}_{\mu}, A q C[\mu] \leq B\right\} \leq \sup \left\{\mathscr{Q}_{A}(C) \wedge \mathscr{2}_{C}(B)\right\}
$$

Hence

$$
\mathscr{2}_{A}(B) \leq \sup _{A q C[\mu] \leq B}\left\{\mathscr{Q}_{A}(C) \wedge \mathscr{2}_{C}(B)\right\}
$$

THEOREM 3.6. If the mapping $2_{A}: \mathscr{A}_{\mu} \rightarrow I$ satisfies the conditions $(\mu Q 1)-(\mu Q 5)$, then the mapping $\mathscr{T}_{\mu}: \mathscr{A}_{\mu} \rightarrow I$, defined by

$$
\mathscr{T}_{\mu}(U)= \begin{cases}\bigwedge_{A q U[\mu]} 2_{A}(U), & U \neq \overline{0} \\ 1, & U=\overline{0}\end{cases}
$$

where $U \in A_{\mu}$, is a smooth $\mu$-topology on $X$.

Proof. It is obvious that $\mathscr{T}_{\mu}(\overline{0})=1$. Using $(\mu \mathrm{Q} 2)$ and $(\mu \mathrm{Q} 5)$ we obtain that $\sup \left\{\mathscr{Q}_{A}(B): B \in \mathscr{A}_{\mu}\right\}=\mathscr{2}_{A}(\mu)=1$, for all $A \in \mathscr{A}_{\mu}$, that is, $\mathscr{T}_{\mu}(\mu)=1$.

For $U_{1}, U_{2} \in \mathscr{A}_{\mu}$, if $U_{1} \wedge U_{2}=\overline{0}$, then it is clear that $\mathscr{T}_{\mu}\left(U_{1} \wedge U_{2}\right)=1 \geq \mathscr{T}_{\mu}\left(U_{1}\right) \wedge \mathscr{T}_{\mu}\left(U_{2}\right)$. Now we assume that $U_{1} \wedge U_{2} \neq \overline{0}$. Since $A q\left(U_{1} \wedge U_{2}\right)[\mu]$ if and only if $A q U_{1}[\mu]$ and 
$A q U_{2}[\mu]$, and applying ( $\left.\mu \mathrm{Q} 3\right)$ we may write

$$
\begin{aligned}
\mathscr{T}_{\mu}\left(U_{1} \wedge U_{2}\right) & =\bigwedge_{A q\left(U_{1} \wedge U_{2}\right)[\mu]} \mathscr{2}_{A}\left(U_{1} \wedge U_{2}\right) \\
& \geq \bigwedge_{A q\left(U_{1} \wedge U_{2}\right)[\mu]}\left[\mathscr{Q}_{A}\left(U_{1}\right) \wedge \mathscr{2}_{A}\left(U_{2}\right)\right] \\
& =\left[\bigwedge_{A q\left(U_{1} \wedge U_{2}\right)[\mu]} \mathscr{2}_{A}\left(U_{1}\right)\right] \wedge\left[\bigwedge_{A q\left(U_{1} \wedge U_{2}\right)[\mu]} \mathscr{2}_{A}\left(U_{2}\right)\right] \\
& \geq\left[\bigwedge_{A q U_{1}[\mu]} \mathscr{2}_{A}\left(U_{1}\right)\right] \wedge\left[\bigwedge_{A q U_{2}[\mu]} \mathscr{2}_{A}\left(U_{2}\right)\right] \\
& =\mathscr{T}_{\mu}\left(U_{1}\right) \wedge \mathscr{T}_{\mu}\left(U_{2}\right) .
\end{aligned}
$$

Let $\left\{U_{i}: i \in J\right\} \subseteq \mathscr{A}_{\mu}$. If $\bigvee_{i \in J} U_{i}=\overline{0}$, then it is obvious that

$$
\mathscr{T}_{\mu}\left(\bigvee_{i \in J} U_{i}\right)=1 \geq \bigwedge_{j \in J} \mathscr{T}_{\mu}\left(U_{i}\right)
$$

Now suppose that $\bigvee_{i \in J} U_{i} \neq \overline{0}$. Considering ( $\left.\mu \mathrm{Q} 4\right)$ and using the fact that $A q\left(\bigvee_{i \in J} U_{i}\right)[\mu]$ if and only if there exists $i_{\circ} \in J$ such that $A q U_{i_{\circ}}[\mu]$ we observe that

$$
\mathscr{2}_{A}\left(\bigvee_{i \in J} U_{i}\right) \geq \mathscr{2}_{A}\left(U_{i_{\circ}}\right) \geq \bigwedge_{A q U_{i_{\circ}}[\mu]} 2_{A}\left(U_{i_{\circ}}\right)=\mathscr{T}_{\mu}\left(U_{i_{\circ}}\right) .
$$

Hence,

$$
\mathscr{T}_{\mu}\left(\bigvee_{i \in J} U_{i}\right)=\bigwedge_{A q\left(\bigvee_{i \in J} U_{i}\right)[\mu]} \mathscr{2}_{A}\left(\bigvee_{i \in J} U_{i}\right) \geq \bigwedge_{i \in J} \mathscr{T}_{\mu}\left(U_{i}\right)
$$

\section{Fuzzy $\mu$-continuity}

DEFINITION 4.1. Let $(X, \mathscr{T})$ and $(Y, \mathcal{U})$ be STSs, $\mu \in I^{X}$, and $f: X \rightarrow Y . f$ is fuzzy $\mu$-continuous if for each $A \in \mathscr{A}_{f(\mu)}, \mathscr{T}_{\mu}\left(\mu \wedge f^{-1}(A)\right) \geq \mathcal{U}_{f(\mu)}(A)$ holds.

REMARK 4.2. Clearly, if $f$ is fuzzy continuous, then it is also fuzzy $\mu$-continuous, but the reciprocal is not in general true as shown by the following example.

EXAMPLE 4.3. Let $X=Y=I$ and $\mu=\overline{0.5}$. Consider the smooth topologies $\mathscr{T}, \mathcal{U}: I^{X} \rightarrow I$ as follows:

$$
\mathscr{T}(A)=\left\{\begin{array}{ll}
1 & \text { if } A=\overline{1}, \overline{0}, \\
0 & \text { otherwise, }
\end{array} \quad U(A)= \begin{cases}1 & \text { if } A=\overline{1}, \overline{0} \\
\frac{1}{2} & \text { if } A=\overline{0.5} \\
0 & \text { otherwise }\end{cases}\right.
$$

Then, the identity mapping $\operatorname{id}_{X}:(X, \mathscr{T}) \rightarrow(X, \mathcal{U})$ is fuzzy $\mu$-continuous. However it is not fuzzy continuous because $1 / 2=\mathcal{U}(\overline{0.5}) \npreceq \mathscr{T}\left(f^{-1}(\overline{0.5})\right)=\mathscr{T}(\overline{0.5})=0$. 
LEMMA 4.4. Let $(X, \mathcal{T})$ and $(Y, \mathcal{U})$ be STSs and $f: X \rightarrow Y$. Let $\left\{\mu_{j}: j \in J\right\} \subset I^{X}$ such that $\bigvee_{j \in J} \mu_{j}=\overline{1}$. Then $f$ is $\mu_{j}$-continuous for each $j \in J$ if and only if $f$ is fuzzy continuous.

Proof. Due to Remark 4.2, it suffices to show that if $f$ is $\mu_{j}$-continuous for each $j \in J$, then $f$ is fuzzy continuous. For each $B \in I^{Y}$ and $j \in J$, we have

$$
\mathscr{T}_{\mu_{j}}\left(f^{-1}(B) \wedge \mu_{j}\right) \geq U_{f\left(\mu_{j}\right)}\left(B \wedge f\left(\mu_{j}\right)\right)
$$

then, $\bigvee\left\{\mathscr{T}(U): U \in I^{X}, U \wedge \mu_{j}=f^{-1}(B) \wedge \mu_{j}\right\} \geq \bigvee\left\{U(V): V \in I^{Y}, V \wedge f\left(\mu_{j}\right)=B \wedge f\left(\mu_{j}\right)\right\}$. By $\bigvee_{j \in J} \mu_{j}=\overline{1}$ we have $U=f^{-1}(B)$ and $V=B$, then

$$
\mathscr{T}\left(f^{-1}(B)\right) \geq \mathcal{U}(B) \quad \forall B \in I^{Y} .
$$

Hence $f$ is fuzzy continuous.

THEOREM 4.5. Let $(X, \mathscr{T})$ and $(Y, \mathcal{U})$ be STSs, $\mu \in I^{X}$, and $f: X \rightarrow Y$ an injective mapping. The following statements are equivalent.

(1) $f$ is fuzzy $\mu$-continuous.

(2) For each $B \in \mathscr{A}_{f(\mu)}, \mathscr{T}_{\mu}\left(\mu-\left(\mu \wedge f^{-1}(B)\right)\right) \geq \mathcal{U}_{f(\mu)}(f(\mu)-B)$.

(3) For each $A \in \mathscr{A}_{\mu}$ and $\alpha \in I_{\circ}, f\left(\mathrm{Cl}_{\mu}(A, \alpha)\right) \leq \mathrm{Cl}_{f(\mu)}(f(A), \alpha)$.

(4) For each $B \in \mathscr{A}_{f(\mu)}$ and $\alpha \in I_{\circ}, \mathrm{Cl}_{\mu}\left(\mu \wedge f^{-1}(B), \alpha\right) \leq f^{-1}\left(\mathrm{Cl}_{f(\mu)}(B, \alpha)\right) \wedge \mu$.

(5) For each $B \in A_{f(\mu)}$ and $\alpha \in I_{\circ}, \mu \wedge f^{-1}\left(\operatorname{Int}_{f(\mu)}(B, \alpha)\right) \leq \operatorname{Int}_{\mu}\left(\mu \wedge f^{-1}(B), \alpha\right)$.

(6) For each $x_{t} \in \mu$ and each $B \in \mathscr{A}_{f(\mu)}, \alpha \in I_{\circ}$ such that $U_{f(\mu)}(B) \geq \alpha$ with $f\left(x_{t}\right) q B[f(\mu)]$, there is $A \in A_{\mu}$ such that $\tau_{\mu}(A) \geq \alpha$ with $x_{t} q A[\mu]$ and $f(A) \leq B$.

(7) For each $x_{t} \in \mu$ and $B \in 2_{f\left(x_{t}\right)}^{\alpha}, \alpha \in I_{\circ}$, there is $A \in 2_{x_{t}}^{\alpha}$ such that $f(A) \leq B$.

(8) For each $x_{t} \in \mu$ and each $B \in 2_{f\left(x_{t}\right)}^{\alpha}, f^{-1}(B) \in 2_{x_{t}}^{\alpha}$.

Proof. $\quad(1) \Rightarrow(2)$. For each $B \in \mathscr{A}_{f(\mu)}$, we have

$$
\begin{aligned}
\mathcal{U}_{f(\mu)}(f(\mu)-B) & \leq \mathcal{T}_{\mu}\left(\mu \wedge f^{-1}(f(\mu)-B)\right) \quad(\text { by }(1)) \\
& =\mathscr{T}_{\mu}\left(\mu \wedge\left(f^{-1} f(\mu)-f^{-1}(B)\right)\right) \\
& =\mathscr{T}_{\mu}\left(\mu-\left(\mu \wedge f^{-1}(B)\right)\right) .
\end{aligned}
$$

(2) $\Rightarrow$ (3). Suppose there exist $A \in \mathscr{A}_{\mu}$ and $\alpha \in I_{\text {o }}$ such that

$$
f\left(\mathrm{Cl}_{\mu}(A, \alpha)\right) \nless \mathrm{Cl}_{f(\mu)}(f(A), \alpha) .
$$

There exist $y \in Y$ and $t \in I_{\circ}$ such that

$$
f\left(\mathrm{Cl}_{\mu}(A, \alpha)\right)(y)>t>\mathrm{Cl}_{f(\mu)}(f(A), \alpha)(y)
$$

If $f^{-1}(\{y\})=\phi$, it is a contradiction because $f\left(\mathrm{Cl}_{\mu}(A, \alpha)\right)(y)=0$. If $f^{-1}(\{y\}) \neq \phi$, there exists $x \in f^{-1}(\{y\})$ such that

$$
f\left(\mathrm{Cl}_{\mu}(A, \alpha)\right)(y) \geq \mathrm{Cl}_{\mu}(A, \alpha)(x)>t>\mathrm{Cl}_{f(\mu)}(f(A), \alpha)(y)
$$


Since $\mathrm{Cl}_{f(\mu)}(f(A), \alpha)(f(x))<t$, there exists $B \in \mathscr{A}_{f(\mu)}$ with $\mathcal{U}_{f(\mu)}(f(\mu)-B) \geq \alpha$ and $f(A) \leq B$ such that

$$
\mathrm{Cl}_{f(\mu)}(f(A), \alpha)(f(x)) \leq B(f(x))<t .
$$

Moreover, $f(A) \leq B$ implies $A \leq f^{-1}(B)$. From (2), $\mathscr{T}_{\mu}\left(\mu-f^{-1}(B)\right)=\mathscr{T}_{\mu}(\mu-(\mu \wedge$ $\left.\left.f^{-1}(B)\right)\right) \geq \bigcup_{f(\mu)}(f(\mu)-B) \geq \alpha$. Thus,

$$
\mathrm{Cl}_{\mu}(A, \alpha)(x) \leq f^{-1}(B)(x)=B(f(x))<t .
$$

This is a contradiction for (4.7).

(3) $\Rightarrow(4)$. For each $B \in \mathscr{A}_{f(\mu)}, \alpha \in I_{\circ}$. Put $A=f^{-1}(B) \wedge \mu$, and from (3), we have

$$
f\left(\mathrm{Cl}_{\mu}\left(\mu \wedge f^{-1}(B), \alpha\right)\right) \leq \mathrm{Cl}_{f(\mu)}\left(f\left(f^{-1}(B) \wedge \mu\right), \alpha\right) \leq \mathrm{Cl}_{f(\mu)}(B \wedge f(\mu), \alpha) .
$$

This implies

$$
\mathrm{Cl}_{\mu}\left(\mu \wedge f^{-1}(B), \alpha\right) \leq f^{-1}\left(f\left(\mathrm{Cl}_{\mu}\left(\mu \wedge f^{-1}(B), \alpha\right)\right)\right) \leq f^{-1}\left(\mathrm{Cl}_{f(\mu)}(B, \alpha)\right) \wedge \mu .
$$

Hence, $\mathrm{Cl}_{\mu}\left(\mu \wedge f^{-1}(B), \alpha\right) \leq f^{-1}\left(\mathrm{Cl}_{f(\mu)}(B, \alpha)\right) \wedge \mu$.

$(4) \Rightarrow(5)$. This is easily proved from Theorem 2.6( $\mu \mathrm{I} 1)$.

$(5) \Rightarrow(1)$. Suppose that $\mathscr{T}_{\mu}\left(\mu \wedge f^{-1}(B)\right) \nsucceq \mathcal{U}_{f(\mu)}(B)$, for each $B \in \mathscr{A}_{f(\mu)}$. Then there exists $\alpha \in I$ 。 such that

$$
\mathscr{T}_{\mu}\left(\mu \wedge f^{-1}(B)\right)<\alpha \leq \mathcal{U}_{f(\mu)}(B)
$$

By Theorem 2.6, $B=\operatorname{Int}_{f(\mu)}(B, \alpha)$. By (5),

$$
\mu \wedge f^{-1}(B)=\mu \wedge f^{-1}\left(\operatorname{Int}_{f(\mu)}(B, \alpha)\right) \leq \operatorname{Int}_{\mu}\left(\mu \wedge f^{-1}(B), \alpha\right) .
$$

On the other hand, by Theorem 2.6( $\mu \mathrm{I} 3)$, we have $\mu \wedge f^{-1}(B) \geq \operatorname{Int}_{\mu}\left(\mu \wedge f^{-1}(B), \alpha\right)$. Thus, $\mu \wedge f^{-1}(B)=\operatorname{Int}_{\mu}\left(\mu \wedge f^{-1}(B), \alpha\right)$, that is, $\mathscr{T}_{\mu}\left(\mu \wedge f^{-1}(B)\right) \geq \alpha$. This is a contradiction for (4.12). Hence $f$ is fuzzy $\mu$-continuous.

$(1) \Rightarrow(6)$. Let $x_{t} \in \mu, \alpha \in I_{\circ}$, and $B \in \mathscr{A}_{f(\mu)}$ such that $\mathcal{u}_{f(\mu)}(B) \geq \alpha$ with $f\left(x_{t}\right) q B[f(\mu)]$. Then, $x_{t} q f^{-1}(B)[\mu]$. By fuzzy $\mu$-continuity of $f$, we have $\mathscr{T}_{\mu}\left(\mu \wedge f^{-1}(B)\right) \geq \mathcal{U}_{f(\mu)}(B) \geq \alpha$ and so, $\mathscr{T}_{\mu}\left(f^{-1}(B)\right) \geq \alpha$. Put $A=f^{-1}(B)$. Then, $\tau_{\mu}(A) \geq \alpha$ and $f(A)=f\left(f^{-1}(B)\right)=B$.

(6) $\Rightarrow(3)$. Let $A \in \mathscr{A}_{\mu}, \alpha \in I_{\circ}, x_{t} \in \mathrm{Cl}_{\mu}(A, \alpha)$, and $B \in \mathscr{A}_{f(\mu)}$ such that $\mathcal{U}_{f(\mu)}(B) \geq \alpha$ with $f\left(x_{t}\right) q B[f(\mu)]$. By (6) there is $C \in \mathscr{A}_{\mu}$ such that $\mathscr{T}_{\mu}(C) \geq \alpha$ with $x_{t} q C[\mu]$ and $f(C) \leq B$. Since $x_{t} \in \mathrm{Cl}_{\mu}(A, \alpha), \mathscr{T}_{\mu}(C) \geq \alpha$, and $x_{t} q C[\mu]$, then by Theorem 2.7, we have $A q C[\mu]$ which implies that $f(A) q f(C)[f(\mu)]$ and hence $f(A) q B[f(\mu)]$. Thus, $f\left(x_{t}\right) \in \mathrm{Cl}_{f(\mu)}(f(A), \alpha)$, and $x_{t} \in f^{-1}\left(\mathrm{Cl}_{f(\mu)}(f(A), \alpha)\right)$ which implies that $\mathrm{Cl}_{\mu}(A, \alpha) \leq$ $f^{-1}\left(\mathrm{Cl}_{f(\mu)}(f(A), \alpha)\right)$. Hence $f\left(\mathrm{Cl}_{\mu}(A, \alpha)\right) \leq \mathrm{Cl}_{f(\mu)}(f(A), \alpha)$.

(6) $\Rightarrow(7)$. Let $x_{t} \in \mu$ and $B \in 2_{f\left(x_{t}\right)}^{\alpha}$. Then there exists $C \in \mathscr{A}_{f(\mu)}$ such that $\boldsymbol{U}_{f(\mu)}(C) \geq \alpha$ and $f\left(x_{t}\right) q C[f(\mu)] \leq B$. By (6) there is $A \in \mathscr{A}_{\mu}$ such that $\mathscr{T}_{\mu}(A) \geq \alpha$ with $x_{t} q A[\mu]$ and $f(A) \leq C \leq B$. Hence, $A \in 2_{x_{t}}^{\alpha}$ and $f(A) \leq B$. 
(7) $\Rightarrow(8)$. Let $x_{t} \in \mu$ and $B \in 2_{f\left(x_{t}\right)}^{\alpha}$. By (7), there is $C \in 2_{x_{t}}^{\alpha}$ such that $f(C) \leq B$. So, there is $A \in \mathscr{A}_{\mu}$ such that $x_{t} q A[\mu]$ and $A \leq C \leq f^{-1}(B)$. Hence $f^{-1}(B) \in 2_{x_{t}}^{\alpha}$.

$(8) \Rightarrow(6)$. Let $x_{t} \in \mu$ and $B \in \mathscr{A}_{f(\mu)}$ such that $U_{f(\mu)}(B) \geq \alpha$ with $f\left(x_{t}\right) q B[f(\mu)]$. Then, $B \in 2_{f\left(x_{t}\right)}^{\alpha}$. By (8), $f^{-1}(B) \in 2_{x_{t}}^{\alpha}$ and hence there is $A \in \mathscr{A}_{\mu}$ such that $\mathscr{T}_{\mu}(A) \geq \alpha$ with $x_{t} q A[\mu] \leq f^{-1}(B)$ and $f(A) \leq B$.

THEOREM 4.6. Let $(X, \mathcal{T}),(Y, \mathcal{U})$, and $(Z, \mathscr{V})$ be STSs, $\mu \in I^{X}, f: X \rightarrow Y$, and $g: Y \rightarrow Z$. If $f$ is fuzzy $\mu$-continuous and $g$ is fuzzy $f(\mu)$-continuous, then $g \circ f$ is fuzzy $\mu$-continuous.

Definition 4.7. Let $(X, \mathcal{T})$ be an STS, $\mu \in I^{X}$, and $A \in \mathscr{A}_{\mu}$. For each $\alpha \in I_{\circ}, A$ is said to be

(1) $\alpha$-fuzzy $\mu$-regular open if and only if $A=\operatorname{Int}_{\mu}\left(\mathrm{Cl}_{\mu}(A, \alpha), \alpha\right)$;

(2) $\alpha$-fuzzy $\mu$-regular closed if and only if $A=\mathrm{Cl}_{\mu}\left(\operatorname{Int}_{\mu}(A, \alpha), \alpha\right)$.

DeFinition 4.8. Let $(X, \mathscr{T})$ and $(Y, \mathcal{U})$ be STSs and let $\mu \in I^{X}, \alpha \in I_{\circ}$. Then, the mapping $f: X \rightarrow Y$ is fuzzy $\mu$-almost continuous if $\mathcal{T}_{\mu}\left(\mu \wedge f^{-1}(A)\right) \geq \alpha$ for each $\alpha$ fuzzy $f(\mu)$-regular open set $A$ in $\mathscr{A}_{f(\mu)}$.

REMARK 4.9. One may notice that, if $f$ is fuzzy almost continuous [8], then $f$ is fuzzy $\mu$-almost continuous, but the converse is not true in general as shown by Example 4.10. Also, if $f$ is fuzzy $\mu$-continuous, then $f$ is fuzzy $\mu$-almost continuous, but the converse is not true in general as shown by Example 4.10.

EXAMPLE 4.10. We consider Example 4.3, and put

$$
\mathscr{V}(A)= \begin{cases}1 & \text { if } A=\overline{0}, \overline{1}, \\ \frac{1}{2} & \text { if } A=\overline{0.5}, \\ \frac{1}{3} & \text { if } A=\overline{0.3}, \\ 0 & \text { otherwise. }\end{cases}
$$

For an STS $(X, \mathscr{V})$ and $\mu=\overline{0.5}$, we have

(1) the identity mapping $\operatorname{id}_{X}:(X, \mathscr{T}) \rightarrow(X, \mathcal{U})$ is fuzzy $\mu$-almost continuous, but not fuzzy almost continuous;

(2) the identity mapping $\operatorname{id}_{X}:(X, \mathscr{T}) \rightarrow(X, \mathscr{V})$ is fuzzy $\mu$-almost continuous, but not fuzzy $\mu$-continuous.

THEOREM 4.11. Let $(X, \mathscr{T})$ and $(Y, \mathcal{U})$ be STSs, $\mu \in I^{X}$, and $f: X \rightarrow Y$ an injective mapping. The following statements are equivalent.

(1) $f$ is fuzzy $\mu$-almost continuous.

(2) For each $\alpha$-fuzzy $f(\mu)$-regular closed $B \in \mathscr{A}_{f(\mu)}, \alpha \in I_{\circ}$, there exists $\mathscr{T}_{\mu}(\mu-(\mu \wedge$ $\left.\left.f^{-1}(B)\right)\right) \geq \alpha$.

(3) For each $B \in \mathscr{A}_{f(\mu)}$ and $\alpha \in I_{\circ}$ such that $\varkappa_{f(\mu)}(B) \geq \alpha$ there exists $f^{-1}(B) \wedge \mu \leq$ $\operatorname{Int}_{\mu}\left(f^{-1}\left(\operatorname{Int}_{f(\mu)}\left(\mathrm{Cl}_{f(\mu)}(B, \alpha), \alpha\right)\right) \wedge \mu, \alpha\right)$.

(4) For each $B \in \mathscr{A}_{f(\mu)}$ and $\alpha \in I_{\circ}$ such that $\boldsymbol{U}_{f(\mu)}(f(\mu)-B) \geq \alpha$ there exists $f^{-1}(B) \wedge$ $\mu \geq \mathrm{Cl}_{\mu}\left(f^{-1}\left(\mathrm{Cl}_{f(\mu)}\left(\operatorname{Int}_{f(\mu)}(B, \alpha), \alpha\right)\right) \wedge \mu, \alpha\right)$. 
(5) For each $x_{t} \in \mu$ and each $B \in \mathscr{A}_{f(\mu)}, \alpha \in I_{\circ}$ such that $\varkappa_{f(\mu)}(B) \geq \alpha$ with $f\left(x_{t}\right) q B[f(\mu)]$, there is $A \in \mathscr{A}_{\mu}$ such that $\mathscr{T}_{\mu}(A) \geq \alpha$ with $x_{t} q A[\mu]$ and $f(A) \leq$ $\operatorname{Int}_{f(\mu)}\left(\mathrm{Cl}_{f(\mu)}(B, \alpha), \alpha\right)$.

(6) For each $x_{t} \in \mu$ and $B \in 2_{f\left(x_{t}\right)}^{\alpha}, \alpha \in I_{\circ}$ there is $A \in 2_{x_{t}}^{\alpha}$ such that $f(A) \leq$ $\operatorname{Int}_{f(\mu)}\left(\mathrm{Cl}_{f(\mu)}(B, \alpha), \alpha\right)$.

(7) For each $x_{t} \in \mu$ and each $B \in 2_{f\left(x_{t}\right)}^{\alpha}$, there exists $f^{-1}\left(\operatorname{Int}_{f(\mu)}\left(\mathrm{Cl}_{f(\mu)}(B, \alpha), \alpha\right)\right) \in 2_{x_{t}}^{\alpha}$.

Proof. $(1) \Rightarrow(2)$ for each $\alpha$-fuzzy $f(\mu)$-regular closed $B$ and $\alpha \in I_{\circ}$. Then $f(\mu)-B$ is $\alpha$-fuzzy $f(\mu)$-regular open. Since $f$ is fuzzy $\mu$-almost continuous, $\mathscr{T}_{\mu}\left(\mu \wedge f^{-1}(f(\mu)-\right.$ $B)) \geq \alpha$ and hence $\mathscr{T}_{\mu}\left(\mu \wedge\left(\mu-f^{-1}(B)\right)\right) \geq \alpha$. Let $A(x)=\left(\mu-\left(f^{-1}(B) \wedge \mu\right)\right)(x)=\mu(x)-$ $\left(f^{-1}(B) \wedge \mu\right)(x)=\mu(x)-\min \left\{f^{-1}(B)(x), \mu(x)\right\}$. If $\mu(x) \leq f^{-1}(B)(x)$, then $A(x)=$ $\mu(x)-\mu(x)=0$ and $\mathscr{T}_{\mu}(A)=1 \geq \alpha$ and hence $\mathscr{T}_{\mu}\left(\mu-\left(f^{-1}(B) \wedge \mu\right)\right) \geq \alpha$. If $\mu(x)>$ $f^{-1}(B)(x)$, then $A=\mu-f^{-1}(B)=\left(\mu-f^{-1}(B)\right) \wedge \mu$ and hence $\mathscr{T}_{\mu}(A)=\mathscr{T}_{\mu}\left(\mu-\left(f^{-1}(B)\right) \wedge\right.$ $\mu) \geq \alpha$. Thus, for each $\alpha$-fuzzy $f(\mu)$-regular closed set $B, \mathscr{T}_{\mu}\left(\mu-\left(f^{-1}(B) \wedge \mu\right)\right) \geq \alpha$.

$(2) \Rightarrow(1)$. It is clear.

$(1) \Rightarrow(3)$. Let $B \in \mathscr{A}_{f(\mu)}, \alpha \in I_{\circ}$ with $\varkappa_{f(\mu)}(B) \geq \alpha$. Then $B \leq \operatorname{Int}_{f(\mu)}\left(\mathrm{Cl}_{f(\mu)}(B, \alpha), \alpha\right)$ and $\operatorname{Int}_{f(\mu)}\left(\mathrm{Cl}_{f(\mu)}(B, \alpha), \alpha\right)$ is $\alpha$-fuzzy $f(\mu)$-regular open. Since $f$ is fuzzy $\mu$-almost continuous,

$$
f^{-1}(B) \wedge \mu \leq f^{-1}\left(\operatorname{Int}_{f(\mu)}\left(\mathrm{Cl}_{f(\mu)}(B, \alpha), \alpha\right)\right) \wedge \mu,
$$

and $\mathscr{T}_{\mu}\left(\mu \wedge f^{-1}\left(\operatorname{Int}_{f(\mu)}\left(\mathrm{Cl}_{f(\mu)}(B, \alpha), \alpha\right)\right)\right) \geq \alpha$. Thus

$$
f^{-1}(B) \wedge \mu \leq \operatorname{Int}_{\mu}\left(f^{-1}\left(\operatorname{Int}_{f(\mu)}\left(\mathrm{Cl}_{f(\mu)}(B, \alpha), \alpha\right)\right) \wedge \mu, \alpha\right) .
$$

(3) $\Rightarrow(4)$. This follows from Theorem 2.6( $\mu \mathrm{I} 1)$.

(4) $\Rightarrow(2)$. Let $B$ be $\alpha$-fuzzy $f(\mu)$-regular closed, $\alpha \in I_{\circ}$. Then by (4),

$$
\begin{aligned}
f^{-1}(B) \wedge \mu & \geq \mathrm{Cl}_{\mu}\left(f^{-1}\left(\mathrm{Cl}_{f(\mu)}\left(\operatorname{Int}_{f(\mu)}(B, \alpha), \alpha\right)\right) \wedge \mu, \alpha\right) \\
& =\mathrm{Cl}_{\mu}\left(f^{-1}(B) \wedge \mu, \alpha\right) .
\end{aligned}
$$

Thus, $\mathscr{T}_{\mu}\left(\mu-\left(\mu \wedge f^{-1}(B)\right)\right) \geq \alpha$ (by Theorem 2.5 $\left.(\mu \mathrm{C} 2)\right)$.

$(1) \Rightarrow(5) \Rightarrow(3)$ and $(5) \Rightarrow(6) \Rightarrow(7) \Rightarrow(5)$ are similar to that of Theorem 4.5 .

\section{Fuzzy $\mu$-separation axioms}

DeFINITION 5.1. Let $(X, \mathscr{T})$ be an STS, $\alpha \in I_{\circ}$, and $\mu \in I^{X} . \mu$ is said to be $\alpha$-fuzzy $\mu$-Hausdorff if for each $x_{t}, y_{s}(x \neq y) \in \mu$, there are $U_{1}, U_{2} \in \mathscr{A}_{\mu}$ such that $\mathscr{T}_{\mu}\left(U_{1}\right) \geq \alpha$ and $\mathscr{T}_{\mu}\left(U_{2}\right) \geq \alpha$ such that $x_{t} \in U_{1}, y_{s} \in U_{2}$, and $U_{1} \phi U_{2}[\mu]$.

THEOREM 5.2. Let $(X, \mathcal{T})$ be an STS, $\alpha \in I_{\circ}$, and $\mu \in I^{X} . \mu$ is $\alpha$-fuzzy $\mu$-Hausdorff if and only if for each $x_{t}, y_{s}(x \neq y) \in \mu, y_{s} \notin\left\{\mathrm{Cl}_{\mu}(U, \alpha): \mathscr{T}_{\mu}(U) \geq \alpha, x_{t} \in U\right\}$. 
Proof. Let $x_{t}, y_{s}(x \neq y) \in \mu$ and $m=\mu(y)-s$. Then $x_{t}, y_{m}(x \neq y) \in \mu$. Since $\mu$ is $\alpha$-fuzzy $\mu$-Hausdorff, $\alpha \in I_{\circ}$, there are $U_{1}, U_{2} \in \mathscr{A}_{\mu}$ with $\mathscr{T}_{\mu}\left(U_{1}\right) \geq \alpha, \mathscr{T}_{\mu}\left(U_{2}\right) \geq \alpha$ such that $x_{t} \in U_{1}, y_{m} \in U_{2}$, and $U_{1} \phi U_{2}[\mu]$ and hence $U_{1} \leq \mu-U_{2}$ and $\mathscr{T}_{\mu}\left(\mu-\left(\mu-U_{2}\right)\right) \geq \alpha$, which implies $\mathrm{Cl}_{\mu}\left(U_{1}, \alpha\right) \leq \mu-U_{2}$. Since $y_{m} \in U_{2}, s=\mu(y)-m>\mu(y)-U_{2}(y) \geq$ $\left(\mathrm{Cl}_{\mu}\left(U_{1}, \alpha\right)\right)(y)$ and hence $y_{s} \notin \mathrm{Cl}_{\mu}\left(U_{1}, \alpha\right)$. Thus, $y_{s} \notin \bigwedge\left\{\mathrm{Cl}_{\mu}\left(U_{1}, \alpha\right): \mathscr{T}_{\mu}\left(U_{1}\right) \geq \alpha\right.$, $\left.x_{t} \in U_{1}\right\}$.

Conversely, let $x_{t}, y_{s}(x \neq y) \in \mu$. Then, $x_{t}, y_{\mu(y)-s}(x \neq y) \in \mu$. By hypothesis, there is $U_{1} \in \mathscr{A}_{\mu}$ with $\mathscr{T}_{\mu}\left(U_{1}\right) \geq \alpha$ such that $x_{t} \in U_{1}$ and $y_{\mu(y)-s} \notin \mathrm{Cl}_{\mu}\left(U_{1}, \alpha\right)$ and hence $\mu(y)-s>\left(\mathrm{Cl}_{\mu}\left(U_{1}, \alpha\right)\right)(y)$ which implies $y_{s} \in \mu-\mathrm{Cl}_{\mu}\left(U_{1}, \alpha\right)=U_{2}$ and $\mathscr{T}_{\mu}\left(U_{2}\right) \geq \alpha$. Since $U_{2}=\mu-\mathrm{Cl}_{\mu}\left(U_{1}, \alpha\right) \leq \mu-U_{1}, U_{1} \phi U_{2}[\mu]$. Therefore $\mu$ is $\alpha$-fuzzy $\mu$-Hausdorff.

DefinITION 5.3. Let $(X, \mathscr{T})$ be an STS, $\alpha \in I_{\circ}$, and $\mu \in I^{X} . \mu$ is said to be $\alpha$-fuzzy $\mu$-regular space if for each $F \in \mathscr{A}_{\mu}$ with $\mathscr{T}_{\mu}(\mu-F) \geq \alpha$ and for each fuzzy point $x_{t} \in \mu$ with $x_{t} \phi F[\mu]$, there are $U_{1}, U_{2} \in \mathscr{A}_{\mu}$ such that $\mathscr{T}_{\mu}\left(U_{1}\right) \geq \alpha$ and $\mathscr{T}_{\mu}\left(U_{2}\right) \geq \alpha$ such that $x_{t} \in U_{1}, F \leq U_{2}$, and $U_{1} \not U_{2}[\mu]$.

EXAMPLE 5.4. Let $X=\{x, y, z\}$ be a set. Define a smooth topology $\mathscr{T}: I^{X} \rightarrow I$ as follows:

$$
\mathscr{T}(U)= \begin{cases}1 & \text { if } U=\overline{0} \text { or } \overline{1} \\ \frac{1}{2} & \text { if } U=\chi_{\{x, y\}}, \\ \frac{1}{2} & \text { if } U=\chi_{\{z\}}, \\ 0 & \text { otherwise. }\end{cases}
$$

Then $\mu=\overline{0.9}$ is $1 / 2$-fuzzy $\mu$-regular space.

THEOREM 5.5. Let $(X, \mathcal{T})$ be an STS, $\alpha \in I_{\circ}$, and $\mu \in I^{X}$. Then the following are equivalent.

(1) $\mu$ is $\alpha$-fuzzy $\mu$-regular.

(2) For each fuzzy point $x_{t} \in \mu$ and for each $U_{1} \in \mathscr{A}_{\mu}$ with $\mathscr{T}_{\mu}\left(U_{1}\right) \geq \alpha, x_{t} \in U_{1}$, there is $U_{2} \in \mathscr{A}_{\mu}$ with $\mathscr{T}_{\mu}\left(U_{2}\right) \geq \alpha$ such that $x_{t} \in U_{2} \leq \mathrm{Cl}_{\mu}\left(U_{2}, \alpha\right) \leq U_{1}$.

(3) For each fuzzy point $x_{t} \in \mu$ and for each $F \in \mathscr{A}_{\mu}$ with $\mathscr{T}_{\mu}(\mu-F) \geq \alpha$ and $x_{t} \phi F[\mu]$, there are $U_{2}, U_{3} \in \mathscr{A}_{\mu}$ with $\mathscr{T}_{\mu}\left(U_{2}\right) \geq \alpha, \mathscr{T}_{\mu}\left(U_{3}\right) \geq \alpha$ such that $x_{t} \in U_{2}, F \leq U_{3}$, and $\mathrm{Cl}_{\mu}\left(U_{2}, \alpha\right) \phi U_{3}[\mu]$.

Proof. (1) $\Rightarrow(2)$. Let $x_{t} \in \mu$ be a fuzzy point and $U_{1} \in \mathscr{A}_{\mu}$ with $\mathscr{T}_{\mu}\left(U_{1}\right) \geq \alpha, x_{t} \in U$. Then, $\mathscr{T}_{\mu}\left(\mu-\left(\mu-U_{1}\right)\right) \geq \alpha$ with $x_{t} \phi\left(\mu-U_{1}\right)[\mu]$. By (1), there are $U_{2}, U_{3} \in \mathscr{A}_{\mu}$ with $\mathscr{T}_{\mu}\left(U_{2}\right) \geq \alpha, \mathcal{T}_{\mu}\left(U_{3}\right) \geq \alpha$ such that $x_{t} \in U_{3}, \mu-U_{1} \leq U_{2}$, and $U_{2} \phi U_{3}[\mu]$. Since $U_{2} \phi U_{3}[\mu]$, $U_{3} \leq \mu-U_{2} \leq U_{1}$ and hence $\mathrm{Cl}_{\mu}\left(U_{3}, \alpha\right) \leq \mu-U_{2} \leq U_{1}$. Thus $x_{t} \in U_{3} \leq \mathrm{Cl}_{\mu}\left(U_{3}, \alpha\right) \leq U_{1}$.

(2) $\Rightarrow(3)$. Let $x_{t} \in \mu$ be a fuzzy point, $\alpha \in I_{\circ}$, and $F \in \mathscr{A}_{\mu}$ with $\mathscr{T}_{\mu}(\mu-F) \geq \alpha, x_{t} \phi F[\mu]$. Then $x_{t} \in \mu-F$. By (2), there is $U_{1} \in \mathscr{A}_{\mu}$ with $\mathscr{T}_{\mu}\left(U_{1}\right) \geq \alpha$ such that $x_{t} \in U_{1} \leq \mathrm{Cl}_{\mu}\left(U_{1}\right.$, $\alpha) \leq \mu-F$. By (2) again, there is $U_{2} \in \mathscr{A}_{\mu}$ with $\mathscr{T}_{\mu}\left(U_{2}\right) \geq \alpha$ such that $x_{t} \in U_{2} \leq$ $\mathrm{Cl}_{\mu}\left(U_{2}, \alpha\right) \leq U_{1} \leq \mathrm{Cl}_{\mu}\left(U_{1}, \alpha\right) \leq \mu-F$. Put $U_{3}=\mu-\mathrm{Cl}_{\mu}\left(U_{1}, \alpha\right)$. Hence there are $U_{2}, U_{3} \in$ $\mathscr{A}_{\mu}$ with $\mathscr{T}_{\mu}\left(U_{2}\right) \geq \alpha, \mathscr{T}_{\mu}\left(U_{3}\right) \geq \alpha$ such that $x_{t} \in U_{2}, F \leq U_{3}$, and $\mathrm{Cl}_{\mu}\left(U_{2}, \alpha\right) \not U_{3}[\mu]$.

$(3) \Rightarrow(1)$. It is clear. 


\section{Fuzzy $\mu$-compactness}

Definition 6.1. Let $(X, \mathscr{T})$ be an STS, $\alpha \in I_{\circ}$, and $\mu \in I^{X}$. Then, $\mu$ is $\alpha$-fuzzy $\mu$ compact (resp., $\alpha$-fuzzy $\mu$-almost compact) if and only if for each family $\left\{U_{i} \in \mathscr{A}_{\mu}\right.$ : $\left.\mathscr{T}_{\mu}\left(U_{i}\right) \geq \alpha, i \in \Gamma\right\}$ such that $\left(\bigvee_{i \in \Gamma} U_{i}\right)(x)=\mu(x)$ for all $x \in X$, there exists a finite index set $\Gamma_{\circ} \subset \Gamma$ such that $\left(\bigvee_{i \in \Gamma_{\circ}} U_{i}\right)(x)=\mu(x)$ (resp., $\left.\left(\bigvee_{i \in \Gamma_{\circ}} C_{\mu}\left(U_{i}, \alpha\right)\right)(x)=\mu(x)\right)$ for all $x \in X$.

It is clear that if $\mu$ is $\alpha$-fuzzy $\mu$-compact, then it is $\alpha$-fuzzy $\mu$-almost compact. But the converse need not be true in general as shown by the following example.

EXAMPLE 6.2. Let $X$ be any nonempty set and let $\mathscr{T}: I^{X} \rightarrow I$ be a smooth topology defined as

$$
\mathscr{T}(U)= \begin{cases}1 & \text { if } U=\overline{0} \text { or } \overline{1} \\ \frac{1}{3} & \text { if } U=\bar{\alpha}, \text { for } 0.4<\alpha<0.8 \\ 0 & \text { otherwise. }\end{cases}
$$

Then, $\mu=\overline{0.8}$ is $1 / 3$-fuzzy $\mu$-almost compact but not $1 / 3$-fuzzy $\mu$-compact.

In order to investigate for the condition under which $\alpha$-fuzzy $\mu$-almost compact is $\alpha$-fuzzy $\mu$-compact, we set the following definition.

DEFINITION 6.3. Let $(X, \mathcal{T})$ be an STS, $\alpha \in I_{\circ}$, and $\mu \in I^{X}$. Then, $\mu$ is $\alpha$-fuzzy $\mu$-regular if and only if for each $U_{1} \in \mathscr{A}_{\mu}$ with $\mathscr{T}_{\mu}\left(U_{1}\right) \geq \alpha$,

$$
U_{1}=\bigvee\left\{U_{2} \in \mathscr{A}_{\mu}: \mathscr{T}_{\mu}\left(U_{2}\right) \geq \alpha, \mathrm{Cl}_{\mu}\left(U_{2}, \alpha\right) \leq U_{1}\right\}
$$

THEOREM 6.4. Let $(X, \mathcal{T})$ be an STS, $\alpha \in I_{\circ}$, and $\mu \in I^{X}$ be $\alpha$-fuzzy $\mu$-regular. Then, $\mu$ is $\alpha$-fuzzy $\mu$-almost compact if and only if $\mu$ is $\alpha$-fuzzy $\mu$-compact.

Proof. Let $\left\{U_{i} \in \mathscr{A}_{\mu}: \mathscr{T}_{\mu}\left(U_{i}\right) \geq \alpha, i \in \Gamma\right\}$ be a family such that $\left(\bigvee_{i \in \Gamma} U_{i}\right)(x)=\mu(x)$ for all $x \in X$. Since, $\mu$ is $\alpha$-fuzzy $\mu$-regular, for each $\mathscr{T}_{\mu}\left(U_{i}\right) \geq \alpha$,

$$
U_{i}=\bigvee_{i_{k} \in K_{i}}\left\{U_{i_{k}} \in \mathscr{A}_{\mu}: \mathcal{T}_{\mu}\left(U_{i_{k}}\right) \geq \alpha, \mathrm{Cl}_{\mu}\left(U_{i_{k}}, \alpha\right) \leq U_{i}\right\}
$$

Hence $\left(\bigvee_{i \in \Gamma}\left(\bigvee_{i_{k} \in K_{i}} U_{i_{k}}\right)\right)(x)=\mu(x)$ for all $x \in X$. Since $\mu$ is $\alpha$-fuzzy $\mu$-almost compact, there exists a finite index $J \times K_{J}$ such that

$$
\left(\bigvee_{i \in J}\left(\bigvee_{i_{k} \in K_{J}} \mathrm{Cl}_{\mu}\left(U_{i_{k}}, \alpha\right)\right)\right)(x)=\mu(x) \quad \forall x \in X
$$

For $i \in J$, since $\left(\bigvee_{i_{k} \in K_{j}} \mathrm{Cl}_{\mu}\left(U_{i_{k}}, \alpha\right)\right) \leq U_{i}$ we have $\left(\bigvee_{i \in J} U_{i}\right)(x)=\mu(x)$ for all $x \in X$. Hence $\mu$ is $\alpha$-fuzzy $\mu$-compact.

Definition 6.5. A collection $\sigma \subset A_{\mu}$ is said to be from a fuzzy $\mu$-filterbasis, if for each finite subcollection $\left\{U_{1}, U_{2}, \ldots, U_{2}\right\}$ of $\sigma,\left(\bigwedge_{i=1}^{n} U_{i}\right)(x)>0$ for some $x \in X$. If $\mathscr{T}_{\mu}(U) \geq \alpha$ for each $U \in \sigma$ and $\alpha \in I_{\circ}$, then $\sigma$ is said to form an $\alpha$-fuzzy $\mu$-filterbasis. 
THEOREM 6.6. Let $(X, \mathcal{T})$ be an STS, $\alpha \in I_{\circ}$, and $\mu \in I^{X} . \mu$ is $\alpha$-fuzzy $\mu$-compact if and only if for each fuzzy $\mu$-filterbasis $\sigma$ in $\mu,\left(\bigwedge_{U \in \sigma} \mathrm{Cl}_{\mu}(U, \alpha)\right)(x)>0$ for some $x \in X$.

Proof. Let $\sigma=\left\{U_{i} \in \mathscr{A}_{\mu}: \mathscr{T}_{\mu}\left(U_{i}\right) \geq \alpha, i \in \Gamma\right\}$ be a family such that $\left(\bigvee_{U_{i} \in \sigma} U_{i}\right)(x)=$ $\mu(x)$ for all $x \in X$, and suppose that for each finite subcollection $\left\{U_{1}, U_{2}, \ldots, U_{n}\right\}$ of $\sigma$, there is $x \in X$ such that $U_{i}(x)<\mu(x)$ for each $i=1,2, \ldots, n$. Then $\mu(x)-U_{i}(x)>0$ for each $i=1,2, \ldots, n$. So, $\bigwedge_{i=1}^{n}\left(\mu(x)-U_{i}(x)\right)>0$ and hence $\left\{\mu-U_{i}: U_{i} \in \sigma\right\}$ forms a fuzzy $\mu$-filterbasis. Then, $\left(\bigwedge_{U_{i} \in \sigma} \mathrm{Cl}_{\mu}\left(\mu-U_{i}, \alpha\right)\right)(x)=\left(\bigwedge_{U_{i} \in \sigma}\left(\mu-U_{i}\right)\right)(x)=0$ for each $x \in X$, which is a contradiction. Hence $\mu$ is $\alpha$-fuzzy $\mu$-compact.

Conversely, suppose that there is fuzzy $\mu$-filterbasis $\sigma$ such that $\left(\bigwedge_{U \in \sigma} \mathrm{Cl}_{\mu}(U\right.$, $\alpha))(x)=0$ for each $x \in X$, so that $\left(\bigvee_{U \in \sigma}\left(\mu-\mathrm{Cl}_{\mu}(U, \alpha)\right)\right)(x)=\mu(x)$ for each $x \in X$ and hence $\mathscr{T}_{\mu}\left(\mu-\mathrm{Cl}_{\mu}(U, \alpha)\right) \geq \alpha$. Since $\mu$ is $\alpha$-fuzzy $\mu$-compact, there is a finite subset $\left\{U_{1}, \ldots, U_{n}\right\}$ (say) and hence $\left(\bigvee_{i=1}^{n}\left(\mu-\mathrm{Cl}_{\mu}\left(U_{i}, \alpha\right)\right)\right)(x)=\mu(x)$ for all $x \in X$, which implies $\left(\bigvee_{i=1}^{n}\left(\mu-U_{i}\right)\right)(x)=\mu(x)$ for all $x \in X$. So that $\left(\bigwedge_{i=1}^{n} U_{i}\right)(x)=0$ for all $x \in X$ which is a contradiction. Therefore $\left(\bigwedge_{U \in \sigma}\left(\mathrm{Cl}_{\mu}\left(U_{i}, \alpha\right)\right)\right)(x)>0$ for each $x \in X$.

THEOREM 6.7. Let $(X, \mathscr{T})$ be an STS, $\alpha \in I_{\circ}$, and $\mu \in I^{X} . \mu$ is $\alpha$-fuzzy $\mu$-almost compact if and only if for each $\alpha$-fuzzy $\mu$-filterbasis $\sigma$ in $\mu,\left(\bigwedge_{U \in \sigma} \mathrm{Cl}_{\mu}(U, \alpha)\right)(x)>0$ for some $x \in X$.

Proof. Let $\sigma=\left\{U_{i} \in \mathscr{A}_{\mu}: \mathscr{T}_{\mu}\left(U_{i}\right) \geq \alpha, i \in \Gamma\right\}$ be a family such that $\left(\bigvee_{U_{i} \in \sigma} U_{i}\right)(x)=$ $\mu(x)$ for all $x \in X$, and suppose that for each finite subcollection $\left\{U_{1}, U_{2}, \ldots, U_{n}\right\}$ of $\sigma,\left(\bigvee_{i=1}^{n} \mathrm{Cl}_{\mu}\left(U_{i}, \alpha\right)\right)(x)<\mu(x)$ for some $x \in X$. Then, $\bigwedge_{i=1}^{n}\left(\mu(x)-\mathrm{Cl}_{\mu}\left(U_{i}, \alpha\right)(x)\right)>0$ for some $x \in X$. Thus, $\beta=\left\{\mu-\mathrm{Cl}_{\mu}\left(U_{i}, \alpha\right): U_{i} \in \sigma\right\}$ forms $\alpha$-fuzzy $\mu$-filterbasis. Since $\left(\bigvee_{U_{i} \in \sigma} U_{i}\right)(x)=\mu(x)$ for all $x \in X$, hence $\left(\bigwedge_{U_{i} \in \sigma} \mathrm{Cl}_{\mu}\left(\mu-\mathrm{Cl}_{\mu}\left(U_{i}, \alpha\right), \alpha\right)\right)(x)=0$ for each $x \in X$, which is a contradiction. Hence $\left(\bigvee_{i=1}^{n} \mathrm{Cl}_{\mu}\left(U_{i}, \alpha\right)\right)(x)=\mu(x)$ for some $x \in X$, and $\mu$ is $\alpha$-fuzzy $\mu$-almost compact.

Conversely, suppose that there is $\alpha$-fuzzy $\mu$-filterbasis $\sigma$ such that $\left(\bigwedge_{U \in \sigma} \mathrm{Cl}_{\mu}(U\right.$, $\alpha))(x)=0$ for each $x \in X$, so that $\left(\bigvee_{U \in \sigma}\left(\mu-\mathrm{Cl}_{\mu}(U, \alpha)\right)\right)(x)=\mu(x)$ for each $x \in X$ and hence $\mathscr{T}_{\mu}\left(\mu-\mathrm{Cl}_{\mu}(U, \alpha)\right) \geq \alpha$. Since $\mu$ is $\alpha$-fuzzy $\mu$-almost compact, there is a finite subfamily $\left\{\mu-\mathrm{Cl}_{\mu}\left(U_{i}, \alpha\right): i=1,2, \ldots, n\right\}$ (say) such that $\left(\bigvee_{i=1}^{n} \mathrm{Cl}_{\mu}\left(\mu-\mathrm{Cl}_{\mu}\left(U_{i}, \alpha\right), \alpha\right)\right)(x)=$ $\mu(x)$ for all $x \in X$, which implies $\left(\bigwedge_{i=1}^{n} \mu-\mathrm{Cl}_{\mu}\left(\mu-\mathrm{Cl}_{\mu}\left(U_{i}, \alpha\right), \alpha\right)\right)(x)=0$ for all $x \in X$. So that $\left(\bigwedge_{i=1}^{n} U_{i}\right)(x)=0$ for all $x \in X$, which is a contradiction. Therefore $\left(\bigwedge_{U \in \sigma}\left(\mathrm{Cl}_{\mu}\left(U_{i}\right.\right.\right.$, $\alpha)))(x)>0$ for each $x \in X$.

THEOREM 6.8. Let $(X, \mathcal{T})$ and $(Y, \mathcal{U})$ be STSs, $\alpha \in I_{\circ}, \mu \in I^{X}$, and $f: X \rightarrow Y$ be $a$ fuzzy $\mu$-continuous bijective mapping. If $\mu$ is $\alpha$-fuzzy $\mu$-compact, then $f(\mu)$ is $\alpha$-fuzzy $f(\mu)$-compact.

Proof. Let $\sigma=\left\{U_{i} \in \mathscr{A}_{f(\mu)}: \mathcal{U}_{f(\mu)}\left(U_{i}\right) \geq \alpha\right\}$ such that $\left(\bigvee_{U_{i} \in \sigma} U_{i}\right)(y)=f(\mu)(y)$ for all $y \in Y$. Since $f$ is fuzzy $\mu$-continuous, then $\mathscr{T}_{\mu}\left(\mu \wedge f^{-1}\left(U_{i}\right)\right) \geq \alpha$ for each $U_{i} \in \sigma$. Since $f$ is injective map, $\left(\bigvee_{U_{i} \in \sigma}\left(\mu \wedge f^{-1}\left(U_{i}\right)\right)\right)(x)=\mu(x)$ for all $x \in X$. Since $\mu$ is $\alpha$-fuzzy $\mu$-compact, there is a finite subfamily $\left\{\mu \wedge f^{-1}\left(U_{i}\right): i=1,2, \ldots, n\right\}$ such that $\left(\bigvee_{i=1}^{n}(\mu \wedge\right.$ $\left.\left.f^{-1}\left(U_{i}\right)\right)\right)(x)=\mu(x)$ for all $x \in X$ and hence $\mu(x)=\left(\mu \wedge \bigvee_{i=1}^{n} f^{-1}\left(U_{i}\right)\right)(x)$ for all $x \in X$ which implies $f\left(\mu \wedge\left(\bigvee_{i=1}^{n} f^{-1}\left(U_{i}\right)\right)\right)=f(\mu)$ and hence $f(\mu) \wedge\left(\bigvee_{i=1}^{n} f f^{-1}\left(U_{i}\right)\right)=f(\mu)$. Since $f$ is bijective, $f(\mu) \wedge\left(\bigvee_{i=1}^{n} U_{i}\right)=f(\mu)$ and hence $\left(\bigvee_{i=1}^{n} U_{i}\right)(y)=f(\mu)(y)$ for all $y \in Y$. Thus $f(\mu)$ is $\alpha$-fuzzy $f(\mu)$-compact. 
TheOREM 6.9. Let $(X, \mathcal{T})$ and $(Y, \mathcal{U})$ be STSs, $\alpha \in I_{\circ}, \mu \in I^{X}$, and $f: X \rightarrow Y$ be a fuzzy $\mu$-continuous bijective mapping. If $\mu$ is $\alpha$-fuzzy $\mu$-almost compact, then $f(\mu)$ is $\alpha$-fuzzy $f(\mu)$-almost compact.

Proof. Let $\sigma=\left\{U_{i} \in \mathscr{A}_{f(\mu)}: \mathscr{U}_{f(\mu)}\left(U_{i}\right) \geq \alpha\right\}$ such that $\left(\bigvee_{U_{i} \in \sigma} U_{i}\right)(y)=f(\mu)(y)$ for all $y \in Y$. Since $f$ is fuzzy $\mu$-continuous, then $\mathscr{T}_{\mu}\left(\mu \wedge f^{-1}\left(U_{i}\right)\right) \geq \alpha$ for each $U_{i}$ $\in \sigma$. Since $f$ is injective map, $\left(\bigvee_{U_{i} \in \sigma}\left(\mu \wedge f^{-1}\left(U_{i}\right)\right)\right)(x)=\mu(x)$ for all $x \in X$. Since $\mu$ is $\alpha$-fuzzy $\mu$-almost compact, there is a finite subfamily $\left\{\mu \wedge f^{-1}\left(U_{i}\right): i=1,2, \ldots, n\right\}$ such that $\left(\bigvee_{i=1}^{n} \mathrm{Cl}_{\mu}\left(\mu \wedge f^{-1}\left(U_{i}\right), \alpha\right)\right)(x)=\mu(x)$ for all $x \in X$, and hence by Theorem 4.5(4), we have $\mu(x)=\left(\bigvee_{i=1}^{n} f^{-1}\left(\mathrm{Cl}_{f(\mu)}\left(U_{i}, \alpha\right)\right) \wedge \mu\right)(x)$ for all $x \in X$ which implies $f\left(\bigvee_{i=1}^{n} f^{-1}\left(\mathrm{Cl}_{f(\mu)}\left(U_{i}, \alpha\right)\right) \wedge \mu\right)=f(\mu)$ and hence $f(\mu) \wedge\left(\bigvee_{i=1}^{n} f f^{-1}\left(\mathrm{Cl}_{f(\mu)}\left(U_{i}, \alpha\right)\right)\right)=$ $f(\mu)$. Since $f$ is bijective, $f(\mu) \wedge\left(\bigvee_{i=1}^{n} \mathrm{Cl}_{f(\mu)}\left(U_{i}, \alpha\right)\right)=f(\mu)$ and hence $\left(\bigvee_{i=1}^{n} \mathrm{Cl}_{f(\mu)}\left(U_{i}\right.\right.$, $\alpha))(y)=f(\mu)(y)$ for all $y \in Y$. Thus $f(\mu)$ is $\alpha$-fuzzy $f(\mu)$-almost compact.

\section{Fuzzy $\mu$-connected sets}

DEFINITION 7.1. Let $(X, \mathcal{T})$ be an STS, $\alpha \in I_{\circ}, \mu \in I^{X}$, and $U_{1}, U_{2} \in \mathscr{A}_{\mu}$. Then, $U_{1}, U_{2}$ are said to be $\alpha$-fuzzy $\mu$-separated if $U_{1} \phi \mathrm{Cl}_{\mu}\left(U_{2}, \alpha\right)[\mu]$ and $U_{2} \not \mathrm{Cl}_{\mu}\left(U_{1}, \alpha\right)[\mu]$.

THEOREM 7.2. Let $U_{1}, U_{2} \in \mathscr{A}_{\mu}$ and $\alpha \in I_{\circ}$. Then,

(1) if $U_{1}$ and $U_{2}$ are $\alpha$-fuzzy $\mu$-separated and $V_{1}, V_{2} \in \mathcal{A}_{\mu}$ such that $\phi \neq V_{1} \leq U_{1}$, $\phi \neq V_{2} \leq U_{2}$, then $V_{1}$ and $V_{2}$ are $\alpha$-fuzzy $\mu$-separated;

(2) if $U_{1} \phi U_{2}[\mu]$, and either $\mathscr{T}_{\mu}\left(U_{1}\right) \geq \alpha, \mathscr{T}_{\mu}\left(U_{2}\right) \geq \alpha$ or $\mathscr{T}_{\mu}\left(\mu-U_{1}\right) \geq \alpha, \mathscr{T}_{\mu}\left(\mu-U_{2}\right) \geq$ $\alpha$, then $U_{1}$ and $U_{2}$ are $\alpha$-fuzzy $\mu$-separated;

(3) if either $\mathscr{T}_{\mu}\left(U_{1}\right) \geq \alpha, \mathscr{T}_{\mu}\left(U_{2}\right) \geq \alpha$ or $\mathscr{T}_{\mu}\left(\mu-U_{1}\right) \geq \alpha, \mathscr{T}_{\mu}\left(\mu-U_{2}\right) \geq \alpha$, then $U_{1} \wedge$ $\left(\mu-U_{2}\right)$ and $U_{2} \wedge\left(\mu-U_{1}\right)$ are $\alpha$-fuzzy $\mu$-separated.

Proof. (1) Since $V_{1} \leq U_{1}$. Then $\mathrm{Cl}_{\mu}\left(V_{1}, \alpha\right) \leq \mathrm{Cl}_{\mu}\left(U_{1}, \alpha\right)$ hence $U_{2} \not \mathrm{Cl}_{\mu}\left(V_{1}, \alpha\right)[\mu]$, which implies $V_{2} \not \mathrm{Cl}_{\mu}\left(U_{1}, \alpha\right)[\mu]$. Thus $V_{1}$ and $V_{2}$ are $\alpha$-fuzzy $\mu$-separated.

(2) Let $\mathscr{T}_{\mu}\left(\mu-U_{1}\right) \geq \alpha, \mathscr{T}_{\mu}\left(\mu-U_{2}\right) \geq \alpha$, and $U_{1} \phi U_{2}[\mu]$. Then $U_{1}=\mathrm{Cl}_{\mu}\left(U_{1}, \alpha\right)$ and $U_{2}=\mathrm{Cl}_{\mu}\left(U_{2}, \alpha\right)$ since $U_{1} \phi U_{2}[\mu], U_{1} \not \mathrm{Cl}_{\mu}\left(U_{2}, \alpha\right)[\mu]$, and $U_{2} \phi \mathrm{Cl}_{\mu}\left(U_{1}, \alpha\right)[\mu]$. Thus $U_{1}$ and $U_{2}$ are $\alpha$-fuzzy $\mu$-separated.

Let $\mathscr{T}_{\mu}\left(U_{1}\right) \geq \alpha, \mathscr{T}_{\mu}\left(U_{2}\right) \geq \alpha$, and $U_{1} \phi U_{2}[\mu]$. Then, $U_{1} \leq \mu-U_{2}$ and hence $\mathrm{Cl}_{\mu}\left(U_{1}, \alpha\right) \leq$ $\mu-U_{2}$ which implies $U_{2} \phi \mathrm{Cl}_{\mu}\left(U_{1}, \alpha\right)[\mu]$. Similarly, $U_{1} \phi \mathrm{Cl}_{\mu}\left(U_{2}, \alpha\right)[\mu]$. Thus, $U_{1}$ and $U_{2}$ are $\alpha$-fuzzy $\mu$-separated.

(3) Let $\mathscr{T}_{\mu}\left(U_{1}\right) \geq \alpha, \mathscr{T}_{\mu}\left(U_{2}\right) \geq \alpha$. Then, $\mathscr{T}_{\mu}\left(\mu-\left(\mu-U_{1}\right)\right) \geq \alpha, \mathscr{T}_{\mu}\left(\mu-\left(\mu-U_{2}\right)\right) \geq \alpha$, and hence $\mathrm{Cl}_{\mu}\left(U_{1} \wedge\left(\mu-U_{2}\right), \alpha\right) \leq \mu-U_{2}$. Thus $\mathrm{Cl}_{\mu}\left(U_{1} \wedge\left(\mu-U_{2}\right), \alpha\right) \phi U_{2}[\mu]$ and hence $\mathrm{Cl}_{\mu}\left(U_{1} \wedge\left(\mu-U_{2}\right), \alpha\right) \phi\left(U_{2} \wedge\left(\mu-U_{1}\right)\right)[\mu]$, since $U_{2} \wedge\left(\mu-U_{1}\right) \leq U_{2}$. Similarly, $\mathrm{Cl}_{\mu}\left(U_{2} \wedge(\mu-\right.$ $\left.\left.U_{1}\right), \alpha\right) \not\left(U_{1} \wedge\left(\mu-U_{2}\right)\right)[\mu]$. Thus $U_{1} \wedge\left(\mu-U_{2}\right)$ and $U_{2} \wedge\left(\mu-U_{1}\right)$ are $\alpha$-fuzzy $\mu$-separated.

Let $\mathscr{T}_{\mu}\left(\mu-U_{1}\right) \geq \alpha, \mathscr{T}_{\mu}\left(\mu-U_{2}\right) \geq \alpha$. Then, $U_{1}=\mathrm{Cl}_{\mu}\left(U_{1}, \alpha\right), U_{2}=\mathrm{Cl}_{\mu}\left(U_{2}, \alpha\right)$, and hence $\mathrm{Cl}_{\mu}\left(U_{2}, \alpha\right) \phi\left(U_{1} \wedge\left(\mu-U_{2}\right)\right)[\mu]$ since $U_{2} \wedge\left(\mu-U_{1}\right) \leq U_{2}, \mathrm{Cl}_{\mu}\left(U_{2} \wedge\left(\mu-U_{1}\right), \alpha\right) \phi\left(U_{1} \wedge(\mu-\right.$ $\left.\left.U_{2}\right)\right)[\mu]$. Similarly, $\mathrm{Cl}_{\mu}\left(U_{1} \wedge\left(\mu-U_{2}\right), \alpha\right) \not\left(U_{2} \wedge\left(\mu-U_{1}\right)\right)[\mu]$. Thus, $U_{1} \wedge\left(\mu-U_{2}\right)$ and $U_{2} \wedge\left(\mu-U_{1}\right)$ are $\alpha$-fuzzy $\mu$-separated.

THEOREM 7.3. Let $(X, \mathscr{T})$ be an STS, $\alpha \in I_{\circ}$, and $\mu \in I^{X}$. Then $U_{1}, U_{2} \in \mathscr{A}_{\mu}$ are $\alpha$-fuzzy $\mu$-separated if and only if there are $V_{1}, V_{2} \in \mathcal{A}_{\mu}$ with $\mathscr{T}_{\mu}\left(V_{1}\right) \geq \alpha$ and $\mathscr{T}_{\mu}\left(V_{2}\right) \geq \alpha$ such that $U_{1} \leq V_{1}, U_{2} \leq V_{2}, U_{1} \phi V_{2}[\mu]$, and $U_{2} \phi V_{1}[\mu]$. 
Proof. Let $U_{1}, U_{2} \in A_{\mu}$ be $\alpha$-fuzzy $\mu$-separated. Then $U_{1} \leq \mu-\mathrm{Cl}_{\mu}\left(U_{2}, \alpha\right)=V_{1}, U_{2} \leq$ $\mu-\mathrm{Cl}_{\mu}\left(U_{1}, \alpha\right)=V_{2}, \mathscr{T}_{\mu}\left(V_{1}\right) \geq \alpha, \mathscr{T}_{\mu}\left(V_{2}\right) \geq \alpha, U_{2} \not V_{1}[\mu]$, and $U_{1} \not V_{2}[\mu]$.

Conversely, let $V_{1}, V_{2} \in \mathscr{A}_{\mu}$ with $\mathscr{T}_{\mu}\left(V_{1}\right) \geq \alpha, \mathscr{T}_{\mu}\left(V_{2}\right) \geq \alpha$ such that $U_{1} \leq V_{1}, U_{2} \leq V_{2}$, $U_{1} \not V_{2}[\mu]$, and $U_{2} \not V_{1}[\mu]$. Then, $\mathscr{T}_{\mu}\left(\mu-\left(\mu-V_{1}\right)\right) \geq \alpha, \mathscr{T}_{\mu}\left(\mu-\left(\mu-V_{2}\right)\right) \geq \alpha$ and hence $\mathrm{Cl}_{\mu}\left(U_{1}, \alpha\right) \leq \mu-V_{2} \leq \mu-U_{2}$ and $\mathrm{Cl}_{\mu}\left(U_{2}, \alpha\right) \leq \mu-V_{1} \leq \mu-U_{1}$. Thus, $\mathrm{Cl}_{\mu}\left(U_{1}, \alpha\right) \not U_{2}[\mu]$ and $\mathrm{Cl}_{\mu}\left(U_{2}, \alpha\right) \not U_{1}[\mu]$. Hence $U_{1}, U_{2}$ are $\alpha$-fuzzy $\mu$-separated.

Definition 7.4. $U \in \mathscr{A}_{\mu}$ is said to be $\alpha$-fuzzy $\mu$-connected if it cannot be expressed as the union of two $\alpha$-fuzzy $\mu$-separated sets.

EXAMPLE 7.5. Let $X=\{a, b, c\}, \mu \in I^{X}$, and $U_{1}, U_{2}, U_{3}, A \in \mathscr{A}_{\mu}$ be defined as

$$
\begin{aligned}
\mu(a) & =0.9, & \mu(b) & =0.8, & \mu(c) & =0.7, \\
U_{1}(a) & =0.5, & U_{1}(b) & =0.2, & U_{1}(c) & =0.6, \\
U_{2}(a) & =0.0, & U_{2}(b) & =0.4, & U_{2}(c) & =0.0, \\
U_{3}(a) & =0.0, & U_{3}(b) & =0.0, & U_{3}(c) & =0.1, \\
A(a) & =0.0, & A(b) & =0.4, & A(c) & =0.1 .
\end{aligned}
$$

Clearly $\mathscr{T}: I^{X} \rightarrow I$, defined as

$$
\mathscr{T}(U)= \begin{cases}1 & \text { if } U=\overline{0} \text { or } \overline{1} \\ \frac{1}{2} & \text { if } U=U_{1} \\ 0 & \text { otherwise }\end{cases}
$$

is a smooth topology on $X$.

(1) We easily show that $\mathrm{Cl}_{\mu}\left(U_{2}, 1 / 2\right)=\mathrm{Cl}_{\mu}\left(U_{3}, 1 / 2\right)=\mu-U_{1}$. So, $U_{2} q \mathrm{Cl}_{\mu}\left(U_{3}, 1 / 2\right)[\mu]$ and $U_{3} \not \mathrm{Cl}_{\mu}\left(U_{2}, 1 / 2\right)[\mu]$. Thus $U_{2}$ and $U_{3}$ are not $1 / 2$-fuzzy $\mu$-separated.

(2) We show that $A$ is $1 / 2$-fuzzy $\mu$-connected. In fact, let $A=A_{1} \vee A_{2}$, where $A_{1}, A_{2} \in$ $A_{\mu}-\{\overline{0}\}$. Then either $A_{1}(b)=0.4$ or $A_{2}(b)=0.4$. If $A_{1}(b)=0.4$, then $\mathrm{Cl}_{\mu}\left(A_{2}, 1 / 2\right)=$ $\mu-U_{1}$. So, $A_{1} q \mathrm{Cl}_{\mu}\left(A_{2}, 1 / 2\right)[\mu]$. If $A_{2}(b)=0.6$, similarly, $A_{2} q \mathrm{Cl}_{\mu}\left(A_{1}, 1 / 2\right)[\mu]$. Thus, $A_{1}$ and $A_{2}$ can not be $1 / 2$-fuzzy $\mu$-separated. Hence $A$ is $1 / 2$-fuzzy $\mu$-connected.

TheOREM 7.6. Let $(X, \mathcal{T})$ and $(Y, \mathcal{U})$ be STSs, $\alpha \in I_{\circ}, \mu \in I^{X}$ and $f: X \rightarrow Y$ be a fuzzy $\mu$-continuous injective mapping. If $U \in A_{\mu}$ is $\alpha$-fuzzy $\mu$-connected, then $f(U)$ is $\alpha$-fuzzy $f(\mu)$-connected.

Proof. Suppose that there are $\alpha$-fuzzy $f(\mu)$-separated sets $U_{1}, U_{2} \in \mathscr{A}_{f(\mu)}$ such that $f(U)=U_{1} \vee U_{2}$. By Theorem 7.3, there are $V_{1}, V_{2} \in \mathscr{A}_{f(\mu)}$ with $u_{f(\mu)}\left(V_{1}\right) \geq \alpha, \boldsymbol{U}_{f(\mu)}\left(V_{2}\right) \geq$ $\alpha$ such that $U_{1} \leq V_{1}, U_{2} \leq V_{2}, U_{1} \not V_{2}[f(\mu)]$, and $U_{2} \phi V_{1}[f(\mu)]$. Since $f$ is fuzzy $\mu$ continuous, $\mathscr{T}_{\mu}\left(\mu \wedge f^{-1}\left(V_{1}\right)\right) \geq \alpha, \mathscr{T}_{\mu}\left(\mu \wedge f^{-1}\left(V_{2}\right)\right) \geq \alpha$. Since $f$ is injective and $V_{1} \leq$ $f(\mu), f^{-1}\left(V_{1}\right) \leq f^{-1}(f(\mu))=\mu$ and hence $f^{-1}\left(U_{1}\right) \leq \mu \wedge f^{-1}\left(V_{1}\right)$. Similarly, $f^{-1}\left(U_{2}\right) \leq$ $\mu \wedge f^{-1}\left(V_{2}\right)$. Since $f$ is injective map, $f^{-1}\left(U_{1}\right)(x)+\left(\mu \wedge f^{-1}\left(V_{2}\right)\right)(x)=U_{1}(f(x))+$ $f^{-1}\left(V_{2}\right)(x)=U_{1}(f(x))+V_{2}(f(x))=U_{1}(y)+V_{2}(y) \leq f(\mu)(y) \leq \mu(x)$ and hence $f^{-1}\left(U_{1}\right) \not \mu \wedge f^{-1}\left(V_{2}\right)[\mu]$. Similarly, $f^{-1}\left(U_{2}\right) \not\left(\mu \wedge f^{-1}\left(V_{1}\right)\right)[\mu]$. From Theorem 7.3, 
$f^{-1}\left(U_{1}\right)$ and $f^{-1}\left(U_{2}\right)$ are $\alpha$-fuzzy $\mu$-separated sets. Since $f$ is injective, $U=f^{-1}(f(U))=$ $f^{-1}\left(U_{1} \vee U_{2}\right)=f^{-1}\left(U_{1}\right) \vee f^{-1}\left(U_{2}\right)$, which is a contradiction with the fact that $U$ is $\alpha$ fuzzy $\mu$-connected. Hence $f(U)$ is $\alpha$-fuzzy $f(\mu)$-connected.

\section{REFERENCES}

[1] S. E. Abbas, Fuzzy super irresolute functions, Int. J. Math. Math. Sci. 2003 (2003), no. 42, 2689-2700.

[2] C. L. Chang, Fuzzy topological spaces, J. Math. Anal. Appl. 24 (1968), 182-190.

[3] K. C. Chattopadhyay, R. N. Hazra, and S. K. Samanta, Gradation of openness: fuzzy topology, Fuzzy Sets and Systems 49 (1992), no. 2, 237-242.

[4] K. C. Chattopadhyay and S. K. Samanta, Fuzzy topology: fuzzy closure operator, fuzzy compactness and fuzzy connectedness, Fuzzy Sets and Systems 54 (1993), no. 2, 207212.

[5] M. K. El Gayyar, E. E. Kerre, and A. A. Ramadan, Almost compactness and near compactness in smooth topological spaces, Fuzzy Sets and Systems 62 (1994), no. 2, 193-202.

[6] U. Höhle and A. P. Šostak, Axiomatic foundations of fixed-basis fuzzy topology, Mathematics of Fuzzy Sets, Handb. Fuzzy Sets Ser., vol. 3, Kluwer Academic Publishers, Massachusetts, 1999, pp. 123-272.

[7] B. Hutton, Products of fuzzy topological spaces, Topology Appl. 11 (1980), no. 1, 59-67.

[8] R. Lowen, Fuzzy topological spaces and fuzzy compactness, J. Math. Anal. Appl. 56 (1976), no. 3, 621-633.

[9] M. Macho Stadler and M. Á. De Prada Vicente, On fuzzy subspaces, Fuzzy Sets and Systems 58 (1993), no. 3, 365-373.

[10] A. A. Ramadan, Smooth topological spaces, Fuzzy Sets and Systems 48 (1992), no. 3, 371375.

[11] A. P. Šostak, On a fuzzy topological structure, Rend. Circ. Mat. Palermo (2) Suppl. 11 (1985), 89-103.

[12] _ On some modifications of fuzzy topology, Mat. Vesnik 41 (1989), no. 1, 51-64.

[13] A. M. Zahran, On fuzzy subspaces, Kyungpook Math. J. 41 (2001), no. 2, 361-369.

S. E. Abbas: Department of Mathematics, Faculty of Science, South Valley University, Sohag 82524, Egypt

E-mail address: sabbas73@yahoo.com 


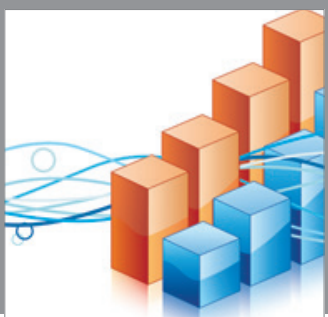

Advances in

Operations Research

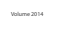

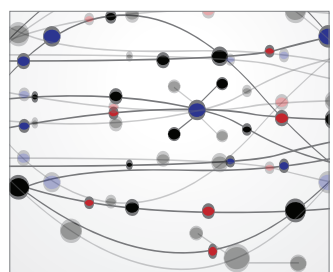

\section{The Scientific} World Journal
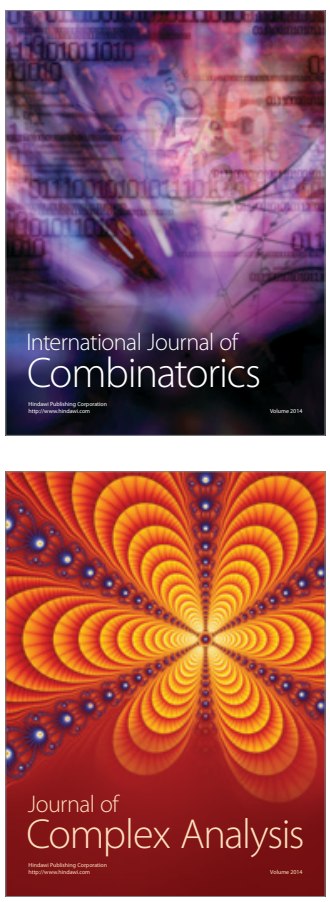

International Journal of

Mathematics and

Mathematical

Sciences
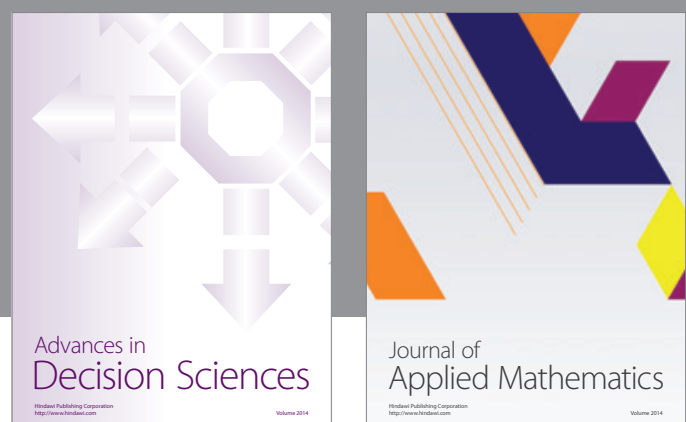

Journal of

Applied Mathematics
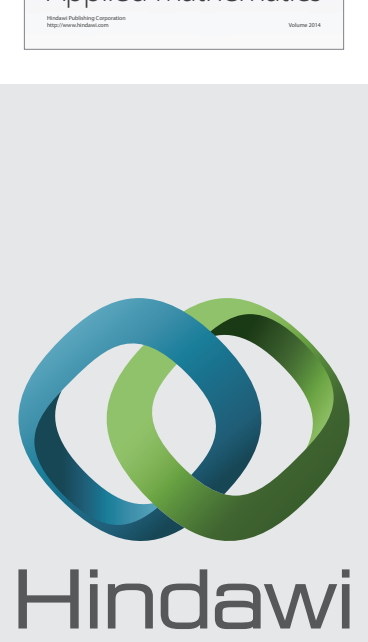

Submit your manuscripts at http://www.hindawi.com
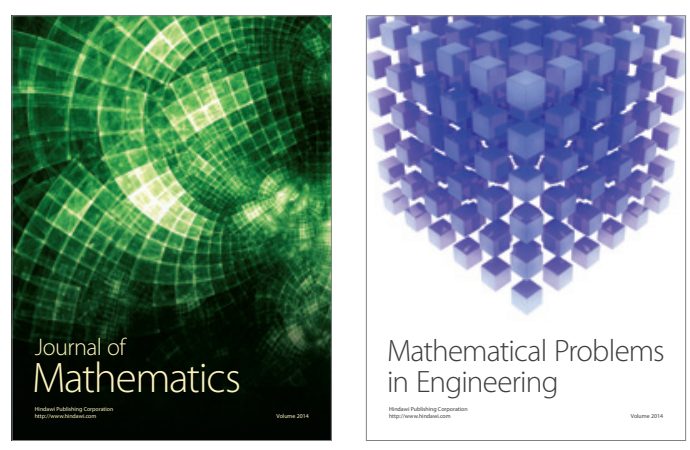

Mathematical Problems in Engineering
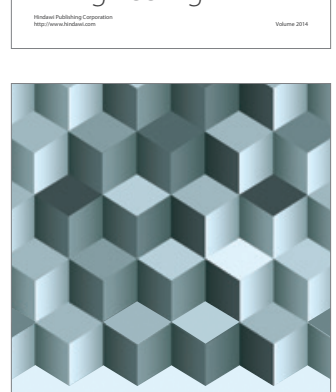

Journal of

Function Spaces
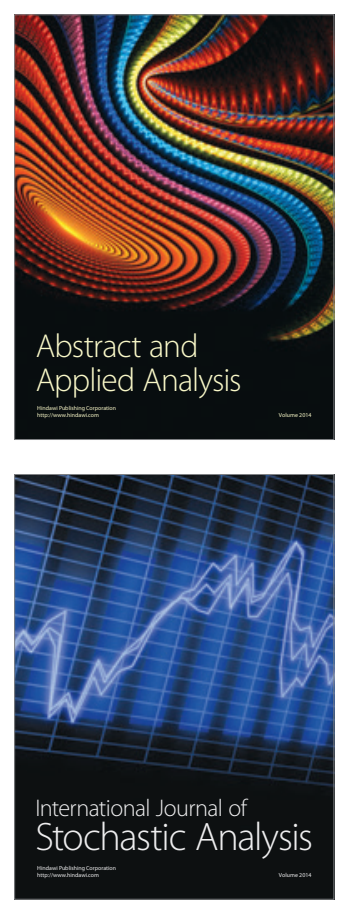

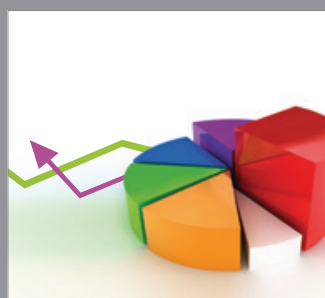

ournal of

Probability and Statistics

Promensencen
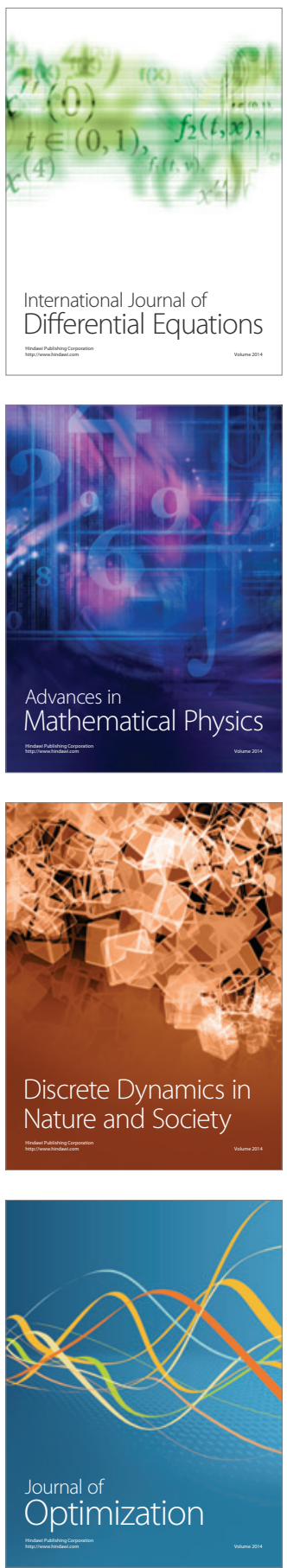